\title{
Annulation of Aromatic Imines via Directed C-H Bond Activation
}

\author{
Reema K. Thalji, Kateri A. Ahrendt, Robert G. Bergman,* Jonathan A. Ellman* \\ Department of Chemistry, University of California and Division of Chemical Sciences, \\ Lawrence Berkeley National Laboratory, Berkeley, CA 94720
}

\section{Supporting Information}

I. General Experimental

II. Preparation and Characterization of Ketone and Aldehyde Precursors

III. Preparation and Characterization of Imine Substrates

S12-S18

IV. Procedure for the Title Reaction and Characterization of Cyclic Products

S18-S25

V. NMR Spectra

S26-S31

VI. References and Footnotes

S32 


\section{General Experimental}

Unless otherwise noted, all reagents were obtained from commercial suppliers and were used without further purification. $\left[\mathrm{RhCl}(\mathrm{coe})_{2}\right]_{2}$ was prepared according to a literature procedure. ${ }^{1}$ All solvents were distilled under $\mathrm{N}_{2}$ from the following drying agents immediately prior to use: $\mathrm{Et}_{2} \mathrm{O}$ and $\mathrm{THF}$ were distilled from $\mathrm{Na} /$ benzophenone ketyl; toluene and $\mathrm{CH}_{2} \mathrm{Cl}_{2}$ were distilled from $\mathrm{CaH}_{2}$. All reactions, unless otherwise noted, were carried out in oven-dried glassware under $\mathrm{N}_{2}$ atmosphere. All C-H activation experiments were assembled in a $\mathrm{N}_{2}$-filled Braun inert atmosphere box. Unless otherwise specified, extracts were dried over $\mathrm{Na}_{2} \mathrm{SO}_{4}$ and solvents were removed with a rotary evaporator using a dry vacuum pump. Flash column chromatography was carried out using Merck 60 230-400 mesh silica gel. Melting points were determined on a MelTemp apparatus and are uncorrected. IR spectra were recorded on a Mattson Galaxy 3000 Fourier Transform spectrometer as thin films on $\mathrm{NaCl}$ plates and only partial data are listed. ${ }^{1} \mathrm{H}$ and ${ }^{13} \mathrm{C}$ NMR spectra were recorded on Bruker DRX-500, AM-400, or AMX-300 instruments, as noted. Unless otherwise noted, ${ }^{1} \mathrm{H}$ and ${ }^{13} \mathrm{C}$ NMR spectra were obtained in $\mathrm{CDCl}_{3}$. NMR chemical shifts are reported in ppm and referenced to the residual protiated solvent signal. Experimental procedures and analytical data for compounds 1-7, ${ }^{2}$ 14-20, ${ }^{2} \mathbf{2 3 - 2 8},{ }^{2} 33-34,{ }^{3} 39-44,{ }^{2} 46,{ }^{3} 48^{3}$, and 53-58 ${ }^{4}$ were reported previously in the referenced publications by our group. 


\section{Preparation and Characterization of Ketone and Aldehyde Precursors}

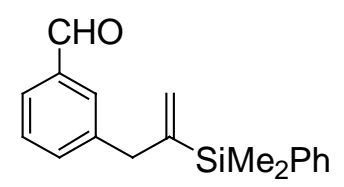

59

3-[2-(Dimethyl-phenyl-silanyl)-allyl]-benzaldehyde (59). $\quad$ To a degassed mixture of (1-bromomethyl-vinyl)-dimethyl-phenyl-silane ${ }^{5}$ (2.00 g, $\left.7.84 \mathrm{mmol}\right), 3-$ formyl-phenylboronic acid (1.76 g, $11.8 \mathrm{mmol})$, toluene $(16 \mathrm{~mL})$, and aqueous $\mathrm{Na}_{2} \mathrm{CO}_{3}$ (8 $\mathrm{mL}$ of a $2 \mathrm{M}$ soln), was added $\mathrm{Pd}\left(\mathrm{PPh}_{3}\right)_{4}(250 \mathrm{mg}, 0.22 \mathrm{mmol})$. The reaction mixture was stirred at $125{ }^{\circ} \mathrm{C}$ for $20 \mathrm{~h}$, diluted with $\mathrm{Et}_{2} \mathrm{O}(100 \mathrm{~mL})$, washed with $\mathrm{H}_{2} \mathrm{O}(50 \mathrm{~mL})$, and then washed with brine $(50 \mathrm{~mL})$. The organic layer was dried, filtered, and concentrated. Silica gel chromatography (2.5\% EtOAc/hex) afforded $\mathbf{5 9}$ as a clear, colorless oil (430 mg, 20\%). IR: 2969, 1697, 1612, 1560, 1480, 1403, 1372, 1324, 1246, 1197, 1060, $975 \mathrm{~cm}^{-1} .{ }^{1} \mathrm{H}$ NMR (400 MHz): $\delta 9.93(\mathrm{~s}, 1 \mathrm{H}), 7.68(\mathrm{~d}, J=7.5 \mathrm{~Hz}, 1 \mathrm{H}), 7.53$ (s, 1H), 7.46-7.30 (m, 7H), 5.61-5.56 (m, 2H), $3.51(\mathrm{~s}, 2 \mathrm{H}), 0.30(\mathrm{~s}, 6 \mathrm{H}),{ }^{13} \mathrm{C}$ NMR $(100$ MHz): $\delta 192.5,149.0,140.9,137.5,136.3,135.5,133.8,130.6,129.1,128.7,128.4$, 127.8, 127.5, 42.3, -3.1. Anal. Calcd. for $\mathrm{C}_{18} \mathrm{H}_{20} \mathrm{OSi}$ C, 77.09; H, 7.19. Found: $\mathrm{C}$, 77.04; H, 7.24.

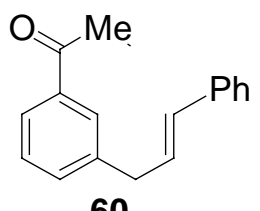

60

1-[3-(3-Phenyl-allyl)-phenyl]-ethanone (60). Magnesium turnings $(0.804 \mathrm{~g}$, $33.1 \mathrm{mmol}$ ) were crushed and flame-dried in a $100 \mathrm{~mL}$ round bottom flask equipped with a pressure equalizing addition funnel. THF $(6 \mathrm{~mL})$ was added to the turnings. At $25{ }^{\circ} \mathrm{C}$, 
approximately 30 drops of a solution of 2-(3-bromo-phenyl)-2-methyl-[1,3]dioxolane ${ }^{6}$ (4.00 g, $16.5 \mathrm{mmol})$ in THF $(10.5 \mathrm{~mL})$ were added to the turnings, and the mixture was stirred until a color change was observed $(\sim 5 \mathrm{~min})$. The remaining solution was then added dropwise over $3 \mathrm{~h}$, and the mixture was stirred for another hour. This Grignard solution was transferred dropwise over 30 min via cannula to a solution of cinnamyl bromide $(4.86 \mathrm{~g}, 24.7 \mathrm{mmol})$ in THF $(24.7 \mathrm{~mL})$. After stirring overnight, the THF was removed in vacuo and the residue was diluted with $\mathrm{Et}_{2} \mathrm{O}$. The resulting solution was washed with $\mathrm{H}_{2} \mathrm{O}$, dried, filtered, and concentrated to a yellow oil. This oil was dissolved in $\mathrm{MeOH}(12 \mathrm{~mL})$, and then $\mathrm{H}_{2} \mathrm{O}(2.4 \mathrm{~mL})$ and conc. aqueous $\mathrm{HCl}(0.75 \mathrm{~mL})$ were added. After stirring for $1 \mathrm{~h}$, the $\mathrm{MeOH}$ was removed in vacuo and the residue was dissolved in $\mathrm{Et}_{2} \mathrm{O}$ and washed once with sat. $\mathrm{NaHCO}_{3}(\mathrm{aq})$. The organic layer was dried, filtered and concentrated to give an orange oil that was purified using $\mathrm{SiO}_{2}$ chromatography (10\% EtOAc/Hex) to provide a light yellow oil $(1.96 \mathrm{~g}, 50 \%)$. IR: 3027, 1680, 1599, 1436, $1271 \mathrm{~cm}^{-1}$. ${ }^{1} \mathrm{H}$ NMR (500 MHz): $\delta 7.85$ (s, 1H), $7.82(\mathrm{~m}, 1 \mathrm{H})$, 7.46-7.20 (m, 6H), $6.48(\mathrm{~d}, J=16 \mathrm{~Hz}, 1 \mathrm{H}), 6.39-6.33(\mathrm{~m}, 1 \mathrm{H}), 3.64(\mathrm{dd}, J=7.0,20.0 \mathrm{~Hz}$, 2H), $2.61(\mathrm{~s}, 3 \mathrm{H}) .{ }^{13} \mathrm{C}\{\mathrm{H}\}$ NMR (100 MHz): $\delta$ 198.2, 140.7, 137.4, 137.2, 133.5, 131.6, 128.7, 128.5, 128.4, 127.3, 126.4, 126.1, 46.7, 26.7. EI-HRMS, Calcd. for $\mathrm{C}_{17} \mathrm{H}_{16} \mathrm{O}[\mathrm{M}]^{+}$ 236.1206; Found, 236.1201.

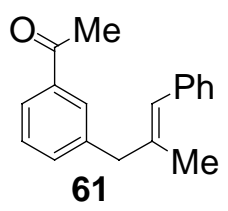

1-[3-(2-Methyl-3-phenyl-allyl)-phenyl]-ethanone (61). This compound was prepared following a procedure similar to that employed for $\mathbf{6 0}$ except that trans-2methyl-cinnamyl bromide (2.61 g, $12.3 \mathrm{mmol})$ was used instead of cinnamyl bromide. 
The crude product was obtained as a 4:1 mixture of the desired ketone $\mathbf{6 1}$ and the undesired regioisomer (the product of $\mathrm{S}_{\mathrm{N}} 2$ ' addition). Silica gel chromatography (10\% EtOAc/Hex) afforded $770 \mathrm{mg}$ (37\% yield) of pure $\mathbf{6 1}$ as a colorless oil (>99\% pure by ${ }^{1} \mathrm{H}$ NMR). IR: $1683,1356,1266,694 \mathrm{~cm}^{-1} . \quad{ }^{1} \mathrm{H}$ NMR (500 MHz): $\delta$ 7.86-7.82 (m, 2H), 7.48-7.19 (m, 7H), $6.39(\mathrm{~s}, 1 \mathrm{H}), 3.54(\mathrm{~s}, 2 \mathrm{H}), 2.62(\mathrm{~s}, 3 \mathrm{H}), 1.80(\mathrm{~s}, 3 \mathrm{H}) .{ }^{13} \mathrm{C}\{\mathrm{H}\} \mathrm{NMR}$ (100 MHz): $\delta$ 198.4, 140.4, 138.1, 137.4, 137.3, 133.8, 128.9, 128.8, 128.6, 128.1, 127.3, 126.5, 126.3, 46.9, 26.8, 17.9. HRMS (EI+) Calcd. for $\mathrm{C}_{18} \mathrm{H}_{18} \mathrm{O}[\mathrm{M}]^{+}$250.1358; Found, 250.1360.

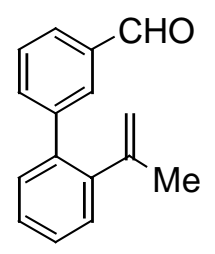

62

2'-Isopropenyl-biphenyl-3-carbaldehyde (62). A mixture of 3-formylphenyl boronic acid (3.00 g, $20.3 \mathrm{mmol}$ ), 1-bromo-2-isopropenyl-benzene ${ }^{7}$ (2.00 g, $10.2 \mathrm{mmol}$ ), $\mathrm{Pd}\left(\mathrm{PPh}_{3}\right)_{4}(586 \mathrm{mg}, 0.510 \mathrm{mmol}), 2 \mathrm{M} \mathrm{NaHCO}_{3}(15 \mathrm{~mL})$, and toluene $(50 \mathrm{~mL})$ was heated under $\mathrm{N}_{2}$ at $100{ }^{\circ} \mathrm{C}$ for $24 \mathrm{~h}$. The reaction mixture was then cooled to room temperature, diluted with EtOAc $(100 \mathrm{~mL})$, washed with $\mathrm{H}_{2} \mathrm{O}(50 \mathrm{~mL})$, and washed with brine $(50 \mathrm{~mL})$. The organic layer was dried, filtered, and concentrated. Silica gel chromatography (2.5\% EtOAc/hexanes) afforded $\mathbf{6 2}$ as a colorless oil (350 mg, 15\%). IR: 1700, 1178, 1164, 898, 758, $697 \mathrm{~cm}^{-1} .{ }^{1} \mathrm{H}$ NMR (400 MHz): $\delta 10.06(\mathrm{~s}, 1 \mathrm{H}), 7.96(\mathrm{t}$, $J=1.7 \mathrm{~Hz}, 1 \mathrm{H}), 7.88-7.85(\mathrm{~m}, 1 \mathrm{H}), 7.74-7.71(\mathrm{~m}, 1 \mathrm{H}), 7.55(\mathrm{t}, J=7.6 \mathrm{~Hz}, 1 \mathrm{H}), 7.39-7.31$ $(\mathrm{m}, 4 \mathrm{H}), 5.12-5.10(\mathrm{~m}, 1 \mathrm{H}), 5.01-4.99(\mathrm{~m}, 1 \mathrm{H}), 1.70(\mathrm{~s}, 3 \mathrm{H}) .{ }^{13} \mathrm{C}$ NMR $(100 \mathrm{MHz}): \delta$ 192.3, 145.7, 143,0, 142.8, 138.0, 136.3, 134.9, 130.3, 130.0, 129.2, 128.7, 128.0, 127.8, 
127.4, 117.0, 23.7. Anal. Calcd. for $\mathrm{C}_{16} \mathrm{H}_{14} \mathrm{O}$ : C, 86.45; H, 6.35. Found: C, 86.60; H, 6.39 .

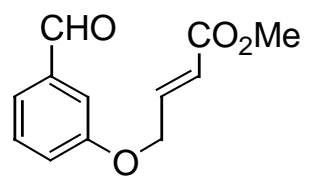

63

4-(3-Formyl-phenoxy)-but-2-enoic acid methyl ester (63). To a mixture of 3hydroxybenzaldehyde (8.53 g, $69.8 \mathrm{mmol}), \mathrm{K}_{2} \mathrm{CO}_{3}(12.5 \mathrm{~g}, 90.8 \mathrm{mmol})$, and DMF (70 $\mathrm{mL})$ was added methyl-3-bromocrotonate $(9.90 \mathrm{~mL}, 83.8 \mathrm{mmol})$. The reaction mixture was stirred at $25{ }^{\circ} \mathrm{C}$ for $14 \mathrm{~h}$, after which it was diluted with EtOAc (300 mL), washed with $\mathrm{H}_{2} \mathrm{O}(3 \times 100 \mathrm{~mL})$, and then washed with brine $(50 \mathrm{~mL})$. The organic layer was dried, filtered, and concentrated. Silica gel chromatography (20\% EtOAc/hexanes) afforded $\mathbf{6 3}$ as a colorless oil (2.10 g, 14\% yield). IR: 2954, 2844, 2734, 1739, 1697 , $1588,1483,1452,1389,1332,1261,1095,1026,792 \mathrm{~cm}^{-1} .{ }^{1} \mathrm{H}$ NMR (400 MHz): $\delta 9.98$ (s, 1H), 7.60-7.56 (m, 1H), 7.52-7.46 (m, 2H), 7.29-7.25 (m, 1H), $6.56(\mathrm{~m}, 1 \mathrm{H})$, 5.17$5.11(\mathrm{~m}, 1 \mathrm{H}), 3.70(\mathrm{~s}, 3 \mathrm{H}), 3.31(\mathrm{dt}, J=7.2,1.6 \mathrm{~Hz}, 2 \mathrm{H}) .{ }^{13} \mathrm{C} \mathrm{NMR}(100 \mathrm{MHz}): \delta 191.5$, $171.9,157.5,141.6,137.9,130.3,124.9,122.7,115.6,105.3,51.9,29.6$. Anal. Calcd. for $\mathrm{C}_{12} \mathrm{H}_{12} \mathrm{O}_{4}$ : C, 65.45; H, 5.49. Found: 65.07; H, 5.56.

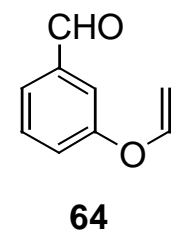

3-Vinyloxy-benzaldehyde (64). To a solution of 3-(2-bromo-ethoxy)benzaldehyde $^{8}(3.00 \mathrm{~g}, 13.1 \mathrm{mmol})$ in DMSO $(13 \mathrm{~mL})$ was added $t$-BuOK $(1.62 \mathrm{~g}, 14.4$ mmol). The reaction mixture was stirred at $25{ }^{\circ} \mathrm{C}$ for $4 \mathrm{~h}$, after which it was diluted with 
EtOAc $(100 \mathrm{~mL})$, washed with $\mathrm{H}_{2} \mathrm{O}(3 \times 50 \mathrm{~mL})$ and then brine $(50 \mathrm{~mL})$. The organic layer was dried, filtered, and concentrated. Silica gel chromatography (5\% EtOAc/hexanes) afforded $\mathbf{6 4}$ as a colorless oil (645 mg, 33\%). IR: 2822, 2735, 1696, $1644,1588,1484,1453,1389,1313,1257,1158 \mathrm{~cm}^{-1} .{ }^{1} \mathrm{H}$ NMR (400 MHz): $\delta 10.01$ (s, 1H), 7.62-7.59 (m, 1H), 7.54-7.49 (m, 2H), 7.31-7.27 (m, 1H), 6.69 (dd, $J=13.7,6.0 \mathrm{~Hz}$, $1 \mathrm{H}), 4.86(\mathrm{dd}, J=13.7,1.8 \mathrm{~Hz}, 1 \mathrm{H}), 4.56(\mathrm{dd}, J=6.0,1.8 \mathrm{~Hz}, 1 \mathrm{H}) .{ }^{13} \mathrm{C}$ NMR $(100$ MHz): $\delta$ 191.5, 157.3, 147.2, 137.9, 130.3, 125.1, 123.3, 116.1, 96.7. EI-HRMS Calcd. for $\mathrm{C}_{9} \mathrm{H}_{8} \mathrm{O}_{2}[\mathrm{M}]^{+}$148.0524; Found, 148.0528.

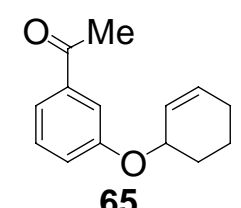

1-[3-(Cyclohex-2-enyloxy)-phenyl]-ethanone (65). To a $250 \mathrm{~mL}$ round bottom flask with a reflux condenser was added 3-hydroxyacetophenone (3.00 g, $22.0 \mathrm{mmol})$, $150 \mathrm{~mL}$ of dry acetone, $\mathrm{K}_{2} \mathrm{CO}_{3}(6.09 \mathrm{~g}, 44.1 \mathrm{mmol})$, and 3-bromocyclohexene (2.79 mL, $24.2 \mathrm{mmol}$ ). The mixture was heated to reflux for $15 \mathrm{~h}$ after which time TLC analysis (10\% EtOAc/Hex) showed quantitative conversion of starting material. The acetone was removed in vacuo and the resulting residue taken up in distilled $\mathrm{H}_{2} \mathrm{O}(100 \mathrm{~mL})$. This was extracted with ether $(3 \times 100 \mathrm{~mL})$ and the combined extracts were dried over $\mathrm{Na}_{2} \mathrm{SO}_{4}$ and concentrated to an orange oil. Purification by chromatography (10\% EtOAc/Hex) provided 65 as a colorless oil (4.05 g, 85\% yield). IR: 2943, 1687, 1591, $1437 \mathrm{~cm}^{-1} .{ }^{1} \mathrm{H}$ NMR (400 MHz): $\delta 7.52-7.50(\mathrm{~m}, 2 \mathrm{H}), 7.35(\mathrm{t}, J=8.1 \mathrm{~Hz}, 1 \mathrm{H}), 7.13-7.11(\mathrm{~m}, 1 \mathrm{H}), 6.00-$ $5.98(\mathrm{~m}, 1 \mathrm{H}), 5.87-5.84(\mathrm{~m}, 1 \mathrm{H}), 4.86(\mathrm{br} \mathrm{s}, 1 \mathrm{H}), 2.58(\mathrm{~s}, 3 \mathrm{H}), 2.11(\mathrm{~m}, 1 \mathrm{H}), 2.05-1.93$ (m, 2H), 1.89-1.83 (m, 2H), $1.66(\mathrm{~m}, 1 \mathrm{H}) .{ }^{13} \mathrm{C}$ NMR (75 MHz): $\delta 198.3,158.4,138.8$, 
132.9, 130.0, 126.1, 121.5, 121.3, 114.9, 71.3, 28.5, 27.1, 25.4, 19.2. Anal. Calcd. for $\mathrm{C}_{14} \mathrm{H}_{16} \mathrm{O}_{2}$ : C, 77.75; H, 7.46. Found: C, 77.65; H, 7.29.

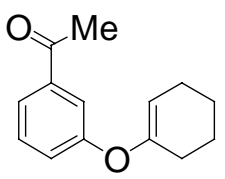

66

1-[3-(Cyclohex-1-enyloxy)-phenyl]-ethanone (66). Ketone 65 (1.00 g, 4.62 $\mathrm{mmol}), \mathrm{RuH}_{2}(\mathrm{CO})\left(\mathrm{PPh}_{3}\right)_{3}(212 \mathrm{mg}, 0.231 \mathrm{mmol})$, and toluene (46 mL) were combined in a glass reaction vessel in an inert atmosphere box. The reaction vessel was sealed with a Kontes stopcock and placed in a $150{ }^{\circ} \mathrm{C}$ oil bath for $68 \mathrm{~h}$. The solvent was removed in vacuo and the residue was chromatographed on silica gel (5\% EtOAc/Hexanes) to afford $370 \mathrm{mg}$ (37\% yield) of $\mathbf{6 6}$ as an oil (>99\% pure by ${ }^{1} \mathrm{H}$ NMR). IR: 1678, 1439, 1264, $1124 \mathrm{~cm}^{-1} .{ }^{1} \mathrm{H}$ NMR (400 MHz): $\delta 7.63(\mathrm{~d}, J=7.6 \mathrm{~Hz}, 1 \mathrm{H}), 7.57(\mathrm{~m}, 1 \mathrm{H}), 7.40(\mathrm{t}, J=8.0$ Hz, 1H), $7.20(\mathrm{~m}, 1 \mathrm{H}), 5.08(\mathrm{t}, J=4.0 \mathrm{~Hz}, 1 \mathrm{H}), 2.60(\mathrm{~s}, 3 \mathrm{H}), 2.20-2.17(\mathrm{~m}, 2 \mathrm{H}), 2.13-$ $2.08(\mathrm{~m}, 2 \mathrm{H}), 1.81-1.75(\mathrm{~m}, 2 \mathrm{H}), 1.66-1.60(\mathrm{~m}, 2 \mathrm{H}) .{ }^{13} \mathrm{C} \mathrm{NMR}(100 \mathrm{MHz}): \delta$ 197.7, $156.7,152.8,138.6,129.6,123.5,122.6,118.1,108.0,26.8,26.5,23.6,22.8,22.1$. EIHRMS Calcd. for $\mathrm{C}_{14} \mathrm{H}_{16} \mathrm{O}_{2}[\mathrm{M}]^{+}:$216.1150; Found, 216.1151.

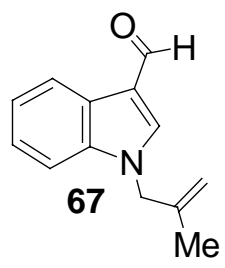

1-(2-Methyl-allyl)-1H-indole-3-carbaldehyde (67). To a mixture of 3carboxyindole (1.00 g, $6.89 \mathrm{mmol}), \mathrm{K}_{2} \mathrm{CO}_{3}(1.90 \mathrm{~g}, 13.8 \mathrm{mmol})$, and dry acetone $(70 \mathrm{~mL})$ was added 3-bromo-2-methylpropene $(0.76 \mathrm{~mL}, 7.58 \mathrm{mmol})$. The reaction mixture was stirred at $25{ }^{\circ} \mathrm{C}$ for $20 \mathrm{~h}$, after which time it was filtered, rinsed with acetone and 
concentrated to an oily residue. This was taken up in $\mathrm{H}_{2} \mathrm{O}(100 \mathrm{~mL})$ and extracted with diethyl ether (3 x $100 \mathrm{~mL})$. The organic layers were combined and then washed with brine $(50 \mathrm{~mL})$, dried, filtered, and concentrated. Silica gel chromatography $(25 \%$ EtOAc/hexanes) afforded 67 as an off-white solid (1.28 g, 94\% yield). MP $37-39{ }^{\circ} \mathrm{C}$. IR: 3105.9, 1658.0, $1531.8 \mathrm{~cm}^{-1}$. ${ }^{1} \mathrm{H}$ NMR (500 MHz): $\delta 10.02(\mathrm{~s}, 1 \mathrm{H}), 8.31(\mathrm{~m}, 1 \mathrm{H}), 7.70$ $(\mathrm{s}, 1 \mathrm{H}), 7.37-7.31(\mathrm{~m}, 3 \mathrm{H}), 5.01(\mathrm{~s}, 1 \mathrm{H}), 4.81(\mathrm{~s}, 1 \mathrm{H}), 4.69(\mathrm{~s}, 2 \mathrm{H}), 1.72(\mathrm{~s}, 3 \mathrm{H}) .{ }^{13} \mathrm{C}$ NMR $(125 \mathrm{MHz}): \delta 184.5,139.5,138.6,137.5,125.3,124.0,122.9,122.0,118.3,114.2$, 110.3, 53.2, 19.8. EI-HRMS Calcd. for $\mathrm{C}_{13} \mathrm{H}_{13} \mathrm{NO}[\mathrm{M}]^{+}$199.0999; Found, 199.0997.

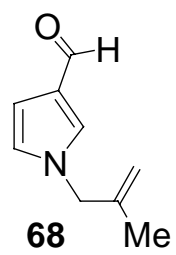

1-(2-Methyl-allyl)-1H-pyrrole-3-carbaldehyde (68). 2-Methylallylamine (0.57 $\mathrm{mL}, 6.2 \mathrm{mmol})$ and acetic acid $(9 \mathrm{~mL})$ were added in succession to stirring 2,5dimethoxytetrahydrofuran-3-carboxaldehyde $(1.00 \mathrm{~g}, 6.24 \mathrm{mmol})$ at $0{ }^{\circ} \mathrm{C}$. This solution was heated to reflux for $2 \mathrm{~h}$ during which time the color turned from yellow to dark brown. After cooling to $25{ }^{\circ} \mathrm{C}$, the solvent was removed in vacuo and the remaining residue was suspended in water $(25 \mathrm{~mL})$. The product was extracted with $\mathrm{CH}_{2} \mathrm{Cl}_{2}(3 \times 25$ $\mathrm{mL})$ and the combined organic portions were dried $\left(\mathrm{Na}_{2} \mathrm{SO}_{4}\right)$, filtered and concentrated to an oil. Silica gel chromatography (50\% EtOAc/Hex) gave $557 \mathrm{mg}(60 \%$ yield) of a viscous orange oil (>99\% pure by ${ }^{1} \mathrm{H}$ NMR). IR: $2768,1664,1156 \mathrm{~cm}^{-1}$. ${ }^{1} \mathrm{H}$ NMR (500 MHz): $\delta 9.74(\mathrm{~s}, 1 \mathrm{H}), 7.27(\mathrm{~m}, 1 \mathrm{H}), 6.63(\mathrm{~m}, 2 \mathrm{H}), 4.97(\mathrm{~m}, 1 \mathrm{H}), 4.81(\mathrm{~m}, 1 \mathrm{H}), 4.42(\mathrm{~s}$ 2H), $1.68(\mathrm{~s}, 3 \mathrm{H}) .{ }^{13} \mathrm{C}$ NMR $(125 \mathrm{MHz}): \delta 185.7,140.9,129.7,127.0,124.1,114.5$, 108.6, 56.7, 20.0. EI-HRMS Calcd. for $\mathrm{C}_{9} \mathrm{H}_{11} \mathrm{NO}[\mathrm{M}]^{+}$149.0843; Found, 149.0841. 


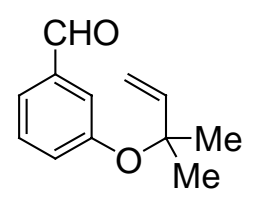

69

3-(1,1-Dimethyl-allyloxy)-benzaldehyde (69). To a solution of 3-(1,1-dimethylprop-2-ynyloxy)-benzaldehyde ${ }^{9}$ (200 mg, $1.06 \mathrm{mmol}$ ), quinoline (6.0 mg, $\left.46 \mu \mathrm{mol}\right)$, and EtOAc $(10 \mathrm{~mL})$ was added Lindlar catalyst $(20 \mathrm{mg}$ of $5 \% \mathrm{Pd} / \mathrm{C})$. The reaction mixture was stirred under a $\mathrm{H}_{2}$ balloon for $4 \mathrm{~h}$, then filtered through Celite ${ }^{\circledR}$ and concentrated. Silica gel chromatography (5\% EtOAc/hexanes) afforded $\mathbf{6 9}$ as a colorless oil (190 mg, 94\%). IR: 2982, 2934, 2724, 1701, 1583, 1481, 1446, 1385, 1261, 1136, 1002, 928, 790, $685 \mathrm{~cm}^{-1} .{ }^{1} \mathrm{H}$ NMR (400 MHz): $\delta 9.94(\mathrm{~s}, 1 \mathrm{H}), 7.52-7.47(\mathrm{~m}, 2 \mathrm{H}), 7.41-7.35(\mathrm{~m}, 1 \mathrm{H})$, 7.27-7.23 (m, 1H), 6.18-6.09 (m, 1H), 5.24-5.16 (m, 2H), $1.49(\mathrm{~s}, 6 \mathrm{H}) .{ }^{13} \mathrm{C}$ NMR (100 MHz): $\delta 192.0,156.7,143.6,137.3,129.4,127.6,123.8,121.1,114.1,80.1,26.9$. Anal. Calcd. for $\mathrm{C}_{12} \mathrm{H}_{14} \mathrm{O}_{2}$ : C, 75.76; H, 7.42. Found: C, 75.57; H, 7.52.

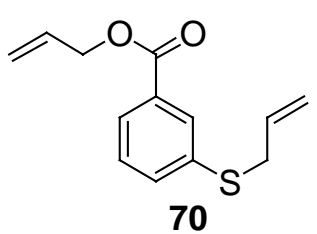

3-Allylsulfanyl-benzoic acid allyl ester (70). To a mixture of 3mercaptobenzoic acid (1.14 g, $7.42 \mathrm{mmol}), \mathrm{K}_{2} \mathrm{CO}_{3}(3.08 \mathrm{~g}, 22.3 \mathrm{mmol})$, and DMF (7.0 $\mathrm{mL}$ ) was added allyl bromide $(1.50 \mathrm{~mL}, 17.8 \mathrm{mmol})$. The reaction mixture was stirred for $30 \mathrm{~min}$, after which time it was diluted with EtOAc $(75 \mathrm{~mL})$, washed with $\mathrm{H}_{2} \mathrm{O}(4 \mathrm{x}$ $50 \mathrm{~mL})$, and then washed with brine $(30 \mathrm{~mL})$. The organic layer was dried, filtered, and concentrated. Silica gel chromatography (7\% EtOAc/hexanes) afforded $\mathbf{7 0}$ as a colorless oil (1.49 g, 86\%). IR: 1722, 1278, 1258, 1126, $748 \mathrm{~cm}^{-1}$. ${ }^{1} \mathrm{H}$ NMR (400 MHz): $\delta 8.04-$ 
$8.02(\mathrm{~m}, 1 \mathrm{H}), 7.89-7.85(\mathrm{~m}, 1 \mathrm{H}), 7.53-7.49(\mathrm{~m}, 1 \mathrm{H}), 7.37-7.33(\mathrm{~m}, 1 \mathrm{H}), 6.10-5.99(\mathrm{~m}$, $1 \mathrm{H})$, 5.93-5.81 (m, 1H), 5.45-5.38 (m, 1H), 5.33-5.28 (m, 1H), 5.20-5.13 (m, 1H), 5.12$5.07(\mathrm{~m}, 1 \mathrm{H}), 4.84-4.80(\mathrm{~m}, 2 \mathrm{H}), 3.61-3.58(\mathrm{~m}, 2 \mathrm{H}) .{ }^{13} \mathrm{C} \mathrm{NMR}(100 \mathrm{MHz}): \delta$ 165.7, 136.7, 133.9, 133.1, 132.1, 130.7, 130.3, 128.7, 127.2, 118.3, 118.0, 65.6, 36.8. Anal. Calcd. for $\mathrm{C}_{13} \mathrm{H}_{14} \mathrm{O}_{2} \mathrm{~S}$ : C, 66.64; H, 6.02; S, 13.68. Found: C, 66.59; H, 6.02; S, 13.31.

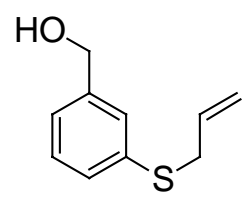

71

(3-Allylsulfanyl-phenyl)-methanol (71). To a solution of 3-allylsulfanylbenzoic acid allyl ester 70, $1.45 \mathrm{~g}, 6.19 \mathrm{mmol})$ in THF $(20 \mathrm{~mL})$ at $0{ }^{\circ} \mathrm{C}$ was added $\mathrm{LiAlH}_{4}$ (517 mg, $13.6 \mathrm{mmol}$ ). The reaction mixture was stirred at $0{ }^{\circ} \mathrm{C}$ for $5 \mathrm{~min}$, then warmed to $25{ }^{\circ} \mathrm{C}$ and stirred an additional $15 \mathrm{~min}$. The reaction mixture was then cooled to $0{ }^{\circ} \mathrm{C}$, and a saturated solution of $\mathrm{Na}_{2} \mathrm{SO}_{4}$ was added dropwise until gas evolution ceased. The mixture was then warmed to room temperature, and stirred vigorously until the grey suspension turned into a clear solution with white precipitate. The solution was diluted with $\mathrm{Et}_{2} \mathrm{O}$, anhydrous $\mathrm{Na}_{2} \mathrm{SO}_{4}$ was added, and the mixture was filtered through Celite®, and then concentrated. Silica gel chromatography (40\% EtOAc/hexanes) afforded $\mathbf{7 1}$ as a colorless oil (1.11 g, 98\%). IR: 3343, 1636, 1593, 1575, 1474, 1426, 1208, 1019, 989, 921, 781, $702 \mathrm{~cm}^{-1} .{ }^{1} \mathrm{H}$ NMR (400 MHz): $\delta$ 7.33-7.30 (m, 1H), 7.28-7.22 (m, 2H), 7.17$7.12(\mathrm{~m}, 1 \mathrm{H}), 5.93-5.82(\mathrm{~m}, 1 \mathrm{H}), 5.19-5.12(\mathrm{~m}, 1 \mathrm{H}), 5.10-5.06(\mathrm{~m}, 1 \mathrm{H}), 4.59(\mathrm{~s}, 2 \mathrm{H})$ 3.58-3.54 (m, 2H), 2.63 (br, 1H). ${ }^{13} \mathrm{C}$ NMR (100 MHz): $\delta 141.5,136.2,133.4,128.9$, 128.5, 127.8, 124.6, 117.7, 64.7, 36.8. Anal. Calcd. for $\mathrm{C}_{10} \mathrm{H}_{12} \mathrm{OS}$ : C, 66.63; H, 6.71; S, 17.79. Found: C, 66.61; H, 6.87; S, 17.57. 


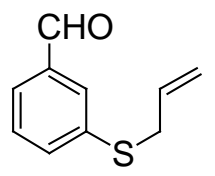

72

3-Allylsulfanyl-benzaldehyde (72). To a solution of (3-allylsulfanyl-phenyl)methanol (71, $150 \mathrm{mg}, 0.830 \mathrm{mmol}), \mathrm{Et}_{3} \mathrm{~N}(0.77 \mathrm{~mL}, 5.50 \mathrm{mmol})$, and DMSO $(0.80 \mathrm{~mL})$ was added $\mathrm{SO}_{3} \cdot$ py $(400 \mathrm{mg}, 2.50 \mathrm{mmol})$. The reaction mixture was stirred for $10 \mathrm{~min}$, then diluted with EtOAc $(20 \mathrm{~mL})$, washed with $\mathrm{H}_{2} \mathrm{O}(2 \times 20 \mathrm{~mL})$, and then washed with brine $(10 \mathrm{~mL})$. The organic layer was dried, filtered, and concentrated. Silica gel chromatography (10\% EtOAc/hexanes) afforded 72 as a colorless oil (122 mg, 82\%). IR: 1698, 1584, 1572, 1199, 923, 786, $682 \mathrm{~cm}^{-1} .{ }^{1} \mathrm{H}$ NMR (400 MHz): $\delta 9.92(\mathrm{~s}, 1 \mathrm{H}), 7.82-$ $7.80(\mathrm{~m}, 1 \mathrm{H}), 7.67-7.65(\mathrm{~m}, 1 \mathrm{H}), 7.58-7.54(\mathrm{~m}, 1 \mathrm{H}), 7.46-7.41(\mathrm{~m}, 1 \mathrm{H})$, 5.93-5.81 (m,

1H), 5.21-5.15 (m, 1H), 5.13-5.08 (m, 1H), 3.63-3.59 (m, 2H). ${ }^{13} \mathrm{C} \mathrm{NMR}(100 \mathrm{MHz}): \delta$ $191.7,137.9,136.8,135.0,132.8,129.6,129.3,127.5,118.2,36.6$. Anal. Calcd. for $\mathrm{C}_{10} \mathrm{H}_{10} \mathrm{OS}$ : C, 67.38; H, 5.65; S, 17.99. Found: C, 67.51; H, 5.73; S, 17.68.

\section{Preparation and Characterization of Imine Substrates}

General procedure for imine synthesis. To a solution of ketone or aldehyde in benzene $(0.5 \mathrm{M})$ was added benzylamine (1.1 equiv). The reaction mixture was stirred over 4A molecular sieves (1.00 g sieves/1.00 mmol substrate) for $12 \mathrm{~h}$, after which it was filtered through Celite ${ }^{\circledR}$ and concentrated in vacuo. Due to its instability, the purity of the crude imine was verified by ${ }^{1} \mathrm{H}$ NMR, and the imine was used without purification in the $\mathrm{C}-\mathrm{H}$ activation experiment within 2-3 days after its isolation. 


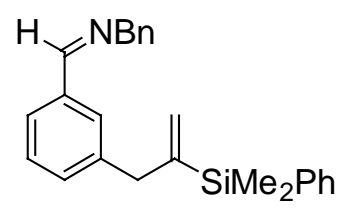

8

Benzyl-\{3-[2-(dimethyl-phenyl-silanyl)-allyl]-benzylidene\}-amine (8). This compound was prepared according to the general procedure for imine formation from $\mathbf{5 9}$ (200 mg, $0.71 \mathrm{mmol})$. Imine 8 was isolated as a yellow oil $(225 \mathrm{mg}, 85 \%) .{ }^{1} \mathrm{H} \mathrm{NMR}$ (400 MHz): $\delta 8.33(\mathrm{~s}, 1 \mathrm{H}), 7.62-7.58(\mathrm{~m}, 1 \mathrm{H}), 7.48-7.44(\mathrm{~m}, 2 \mathrm{H}), 7.37-7.25(\mathrm{~m}, 10 \mathrm{H})$, 7.15-7.11 (m, 1H), 5.56-5.50 (m, 2H), $4.83(\mathrm{~s}, 2 \mathrm{H}), 3.46(\mathrm{~s}, 2 \mathrm{H}), 0.29(\mathrm{~s}, 6 \mathrm{H})$.

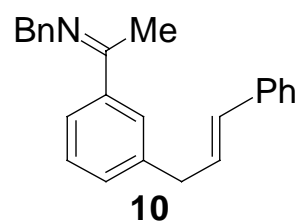

Benzyl-\{1-[3-(3-phenyl-allyl)-phenyl]-ethylidene\}-amine (10). This compound was prepared according to the general procedure for imine formation from $\mathbf{6 0}(500 \mathrm{mg}$, $2.12 \mathrm{mmol}$ ). Imine $\mathbf{1 0}$ was isolated as a light yellow solid after recrystallization from pentane/ $\mathrm{Et}_{2} \mathrm{O}$ in $56 \%$ yield $(380 \mathrm{mg}) . \quad E$ isomer: ${ }^{1} \mathrm{H}$ NMR $(400 \mathrm{MHz}): \delta 7.76(\mathrm{~s}, 1 \mathrm{H})$, $7.70(\mathrm{~m}, 1 \mathrm{H}), 7.44-7.20(\mathrm{~m}, 12 \mathrm{H}), 6.47(\mathrm{~d}, J=12.8 \mathrm{~Hz}, 1 \mathrm{H}), 6.41-6.35(\mathrm{~m}, 1 \mathrm{H}), 4.75(\mathrm{~s}$, 2H), $3.60(\mathrm{~d}, J=5.6 \mathrm{~Hz}, 2 \mathrm{H}), 2.34(\mathrm{~s}, 3 \mathrm{H})$.

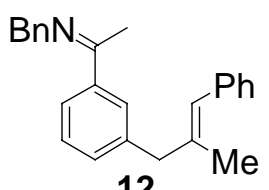

Benzyl-\{1-[3-(2-methyl-3-phenyl-allyl)-phenyl]-ethylidene\}-amine (12). This compound was prepared according to the general procedure for imine formation from $\mathbf{6 1}$ (300 mg, $1.20 \mathrm{mmol}$ ) to afford 12 as a yellow oil in $91 \%$ yield $(368 \mathrm{mg}) . \quad E$ isomer: ${ }^{1} \mathrm{H}$ 
NMR (300 MHz) $\delta$ 7.77-7.71 (m, 2H), 7.46-7.20 (m, 12H), $6.40(\mathrm{~s}, 1 \mathrm{H}), 4.76(\mathrm{~s}, 2 \mathrm{H})$, $3.53(\mathrm{~s}, 2 \mathrm{H}), 2.35(\mathrm{~s}, 3 \mathrm{H}), 1.82(\mathrm{~s}, 3 \mathrm{H})$.

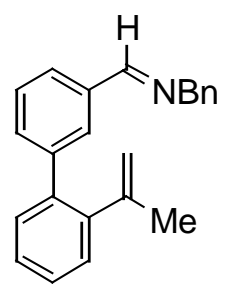

21

Benzyl-(2'-isopropenyl-biphenyl-3-ylmethylene)-amine (21). This compound was prepared according to the general procedure for imine formation from aldehyde $\mathbf{6 2}$ (159 mg, $0.71 \mathrm{mmol})$. Imine 21 was isolated as a colorless oil (200 mg, 90\%). ${ }^{1} \mathrm{H}$ NMR (400 MHz): $\delta 8.43(\mathrm{~s}, 1 \mathrm{H}), 7.83-7.77(\mathrm{~m}, 2 \mathrm{H}), 7.53-7.50(\mathrm{~m}, 1 \mathrm{H}), 7.42(\mathrm{t}, J=7.6 \mathrm{~Hz}$, 1H), 7.38-7.25 (m, 9H), 5.10-5.08 (m, 1H), 5.01-5.00 (m, 1H), $4.86(\mathrm{~s}, 2 \mathrm{H}), 1.69(\mathrm{~s}, 3 \mathrm{H})$.

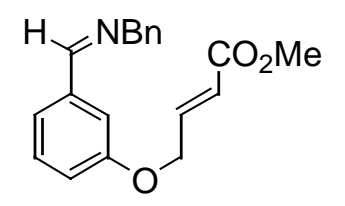

29

4-[3-(Benzylimino-methyl)-phenoxy]-but-2-enoic acid methyl ester (29). This compound was prepared according to the general procedure for imine formation from $\mathbf{6 3}$ (600 mg, $273 \mathrm{mmol})$. Imine 29 was isolated as a light yellow oil $(740 \mathrm{mg}, 88 \%) .{ }^{1} \mathrm{H}$ NMR (400 MHz): $\delta 8.37$ (s, 1H), 7.50-7.26 (m, 8H), 7.10-7.07 (m, 1H), $6.58(\mathrm{dt}, J=6.1$, $1.6 \mathrm{~Hz}, 1 \mathrm{H}), 5.09-5.04(\mathrm{~m}, 1 \mathrm{H}), 4.84(\mathrm{~s}, 2 \mathrm{H}), 3.71(\mathrm{~s}, 3 \mathrm{H}), 3.31(\mathrm{dd}, J=7.1,1.6 \mathrm{~Hz}, 2 \mathrm{H})$. 


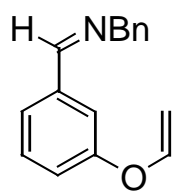

31

Benzyl-(3-vinyloxy-benzylidene)-amine (31). This compound was prepared according to the general procedure for imine formation from aldehyde $\mathbf{6 4}(511 \mathrm{mg}, 3.45$ mmol). Imine 31 was isolated as a light yellow oil (720 mg, 88\%). ${ }^{1} \mathrm{H}$ NMR (400 MHz): $\delta 8.38(\mathrm{~s}, 1 \mathrm{H}), 7.50-7.28(\mathrm{~m}, 8 \mathrm{H}), 7.10-7.07(\mathrm{~m}, 1 \mathrm{H}), 6.70(\mathrm{dd}, J=13.7,6.1 \mathrm{~Hz}, 1 \mathrm{H})$, $4.84(\mathrm{~s}, 2 \mathrm{H}), 4.80(\mathrm{dd}, J=13.7,1.7 \mathrm{~Hz}, 1 \mathrm{H}), 4.47(\mathrm{dd}, J=6.1,1.7 \mathrm{~Hz}, 1 \mathrm{H})$.

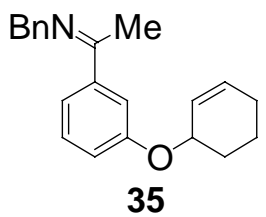

Benzyl-\{1-[3-(cyclohex-2-enyloxy)-phenyl]-ethylidene\}-amine (35). This compound was prepared according to the general procedure for imine formation from $\mathbf{6 5}(1.00 \mathrm{~g}$, $4.62 \mathrm{mmol})$. Imine 35 was isolated as a yellow oil in $98 \%$ yield (1.38 g) as a mixture of $E$ and $Z$ isomers (29:1 by ${ }^{1} \mathrm{H}$ NMR). $E$ isomer: ${ }^{1} \mathrm{H}$ NMR $(400 \mathrm{MHz}) \delta 7.48-7.25(\mathrm{~m}, 8 \mathrm{H})$, 6.98-6.95 (m, 1H), 5.99-5.96 (m, 1H), 5.90-5.87 (m, 1H), 4.86 (br s, 1H), 4.73 (s, 2H), $2.31(\mathrm{~s}, 3 \mathrm{H}), 2.12-1.83(\mathrm{~m}, 5 \mathrm{H}), 1.66(\mathrm{~m}, 1 \mathrm{H}) . \quad Z$ isomer: ${ }^{1} \mathrm{H}$ NMR $(400 \mathrm{MHz}) \delta 7.48-$ $7.25(\mathrm{~m}, 8 \mathrm{H}), 6.89(\mathrm{~m}, 1 \mathrm{H}), 5.99-5.96(\mathrm{~m}, 1 \mathrm{H}), 5.90-5.87(\mathrm{~m}, 1 \mathrm{H}), 4.86(\mathrm{br} \mathrm{s}, 1 \mathrm{H}), 4.44$ (s, 2H), $2.36(\mathrm{~s}, 3 \mathrm{H}), 2.12-1.83(\mathrm{~m}, 5 \mathrm{H}), 1.66(\mathrm{~m}, 1 \mathrm{H})$.

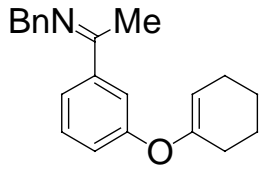

38

Benzyl-\{1-[3-(cyclohex-1-enyloxy)-phenyl]-ethylidene\}-amine (38). This compound was prepared according to the general procedure for imine formation from $\mathbf{6 6}(500 \mathrm{mg}$, 
$2.31 \mathrm{mmol})$. Imine $\mathbf{3 8}$ was isolated as a yellow oil in $98 \%$ yield $(690 \mathrm{mg})$ as a mixture of $E$ and $Z$ isomers (9:1 by ${ }^{1} \mathrm{H}$ NMR). ${ }^{1} \mathrm{H}$ NMR (400 MHz): $\delta$ 7.56-6.80 (m, 9H), 5.13 (m, 0.1H), $5.06(\mathrm{~m}, 0.9 \mathrm{H}), 4.75(\mathrm{~s}, 1.8 \mathrm{H}), 4.46(\mathrm{~s}, 0.2 \mathrm{H}), 2.40(\mathrm{~s}, 0.3 \mathrm{H}), 2.33(\mathrm{~s}, 2.7 \mathrm{H}), 2.22-$ $2.19(\mathrm{~m}, 2 \mathrm{H}), 2.11-2.08(\mathrm{~m}, 2 \mathrm{H}), 1.79-1.74(\mathrm{~m}, 2 \mathrm{H}), 1.64-1.61(\mathrm{~m}, 2 \mathrm{H})$.

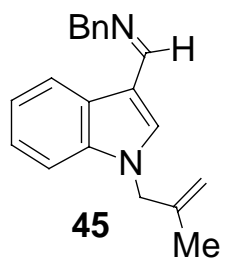

Benzyl-[1-(2-methyl-allyl)-1H-indol-3-ylmethylene]-amine (45). This compound was prepared according to the general procedure for imine formation from 67 (500 mg, $2.51 \mathrm{mmol}$ ). Imine $\mathbf{4 5}$ was obtained as a colorless viscous oil that solidified upon standing (703 mg, 97\% yield). ${ }^{1} \mathrm{H}$ NMR (500 MHz): $\delta 8.60(\mathrm{~m}, 1 \mathrm{H}), 8.39(\mathrm{~m}, 1 \mathrm{H})$, 7.45-7.24 (m, 9H), $4.96(\mathrm{~s}, 1 \mathrm{H}), 4.85(\mathrm{~s}, 2 \mathrm{H}), 4.79(\mathrm{~s}, 1 \mathrm{H}), 4.66(\mathrm{~s}, 2 \mathrm{H}), 1.70(\mathrm{~s}, 3 \mathrm{H})$.

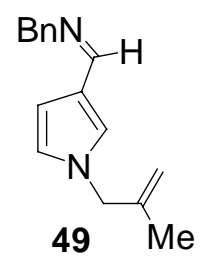

Benzyl-[1-(2-methyl-allyl)-1H-pyrrol-3-ylmethylene]-amine (49). This compound was prepared according to the general procedure for imine formation from 68 (400 mg, $2.68 \mathrm{mmol}$ ). Imine 49 was obtained as an orange viscous oil (635 mg, 99\% yield). ${ }^{1} \mathrm{H}$ NMR (400 MHz): $\delta 8.26(\mathrm{~s}, 1 \mathrm{H}), 7.38-7.22(\mathrm{~m}, 5 \mathrm{H}), 7.03(\mathrm{~m}, 1 \mathrm{H}), 6.63-6.59$ (m, 2H), $4.93(\mathrm{~s}, 1 \mathrm{H}), 4.81(\mathrm{~s}, 1 \mathrm{H}), 4.72(\mathrm{~s}, 2 \mathrm{H}), 4.38(\mathrm{~s}, 2 \mathrm{H}), 1.68(\mathrm{~s}, 3 \mathrm{H})$. 


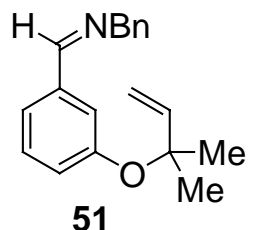

Benzyl-[3-(1,1-dimethyl-allyloxy)-benzylidene]-amine (51). This compound was prepared according to the general procedure for imine formation from aldehyde 69 (91 mg, $0.47 \mathrm{mmol})$. Imine 51 was isolated as a colorless oil $(116 \mathrm{mg}, 88 \%) .{ }^{1} \mathrm{H}$ NMR (400 MHz): $\delta 8.32(\mathrm{~s}, 1 \mathrm{H}), 7.58-7.07(\mathrm{~m}, 9 \mathrm{H}), 6.21-6.12(\mathrm{~m}, 1 \mathrm{H}), 5.23-5.15(\mathrm{~m}, 2 \mathrm{H})$, $4.83(\mathrm{~s}, 2 \mathrm{H}), 1.47(\mathrm{~s}, 6 \mathrm{H})$.

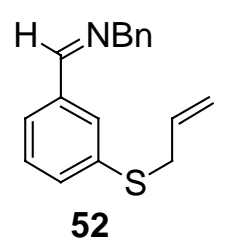

(3-Allylsulfanyl-benzylidene)-benzyl-amine (52). This compound was prepared according to the general procedure for imine formation from aldehyde $\mathbf{7 2}(75 \mathrm{mg}, 0.420$ mmol). Imine 52 was isolated as a colorless oil (100 mg, 89\%). ${ }^{1} \mathrm{H}$ NMR (400 MHz): $\delta 8.34(\mathrm{~s}, 1 \mathrm{H}), 7.79-7.77(\mathrm{~m}, 1 \mathrm{H})$, 7.60-7.56 (m, 1H), 7.42-7.23 (m, 7H), 5.92-5.81 (m, $1 \mathrm{H}), 5.20-5.05(\mathrm{~m}, 2 \mathrm{H}), 4.81(\mathrm{~s}, 2 \mathrm{H}), 3.61-3.56(\mathrm{~m}, 2 \mathrm{H})$.

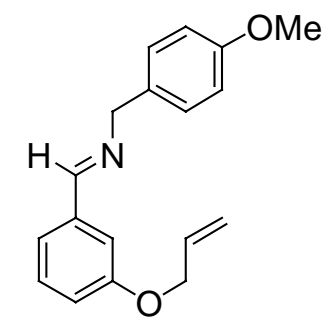

73

(3-Allyloxy-benzylidene)-(4-methoxy-benzyl)-amine (73). This compound was prepared according to the general procedure for imine formation from 3-allyloxybenzaldehyde $^{10}$ (395 mg, $2.4 \mathrm{mmol}$ ). Imine 73 was isolated as a colorless oil (630 mg, 
92\%). ${ }^{1} \mathrm{H}$ NMR (400 MHz): $\delta 8.31(\mathrm{~s}, 1 \mathrm{H}), 7.48-7.13(\mathrm{~m}, 5 \mathrm{H}), 7.04-7.00(\mathrm{~m}, 1 \mathrm{H}), 6.91-$

$6.88(\mathrm{~m}, 2 \mathrm{H}), 6.12-6.01(\mathrm{~m}, 1 \mathrm{H}), 5.56-5.50(\mathrm{~m}, 1 \mathrm{H}), 5.32-5.28(\mathrm{~m}, 1 \mathrm{H}), 4.77(\mathrm{~s}, 2 \mathrm{H})$ 4.61-4.55 (m, 2H), $3.80(\mathrm{~s}, 3 \mathrm{H})$.

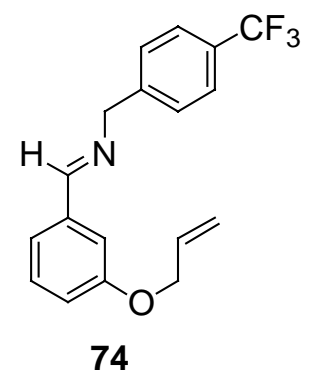

(3-Allyloxy-benzylidene)-(4-trifluoromethyl-benzyl)-amine (74). This compound was prepared according to the general procedure for imine formation from 3allyloxy-benzaldehyde ${ }^{10}$ (395 mg, $2.44 \mathrm{mmol}$ ). Imine $\mathbf{7 4}$ was isolated as a colorless oil (740 mg, 95\%). ${ }^{1} \mathrm{H}$ NMR (400 MHz): $\delta 8.38(\mathrm{~s}, 1 \mathrm{H}), 7.64-7.60(\mathrm{~m}, 2 \mathrm{H}), 7.50-7.42(\mathrm{~m}$, $3 \mathrm{H}), 7.35-7.30(\mathrm{~m}, 2 \mathrm{H}), 7.06-7.01(\mathrm{~m}, 1 \mathrm{H}), 6.12-6.01(\mathrm{~m}, 1 \mathrm{H}), 5.50-5.47(\mathrm{~m}, 1 \mathrm{H})$, 5.33$5.29(\mathrm{~m}, 1 \mathrm{H}), 4.89(\mathrm{~s}, 2 \mathrm{H}), 4.62-4.59(\mathrm{~m}, 2 \mathrm{H})$.

\section{Procedure for the Title Reaction and Characterization of Cyclic Products \\ General procedure for cyclization of benzyl imino substrates. In an inert} atmosphere dry-box, to a medium-walled glass reaction vessel was added a solution of $\left(\mathrm{PPh}_{3}\right)_{3} \mathrm{RhCl}(5 \mathrm{~mol} \%)$ and the benzyl imine in toluene $(0.1 \mathrm{M})$. The vessel was sealed with a Kontes stopcock and placed in an oil bath at $125{ }^{\circ} \mathrm{C}$ or $150{ }^{\circ} \mathrm{C}$. After the noted reaction time, the vessel was removed and cooled to room temperature. The solution was concentrated, and $1 \mathrm{~N} \mathrm{HCl}$ was then added and the resulting mixture vigorously stirred for $3 \mathrm{~h}$. The mixture was then diluted with EtOAc, the organic layer separated, and the aqueous phase re-extracted. The combined organic extracts were washed with brine, 
dried, filtered, and concentrated in vacuo. The crude product was purified by silica gel chromatography.

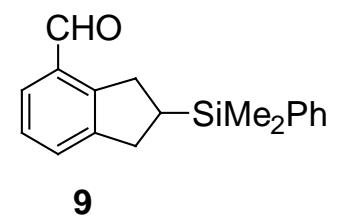

2-(Dimethyl-phenyl-silanyl)-indan-4-carbaldehyde (9). Reaction of 8 (210 mg, $0.568 \mathrm{mmol})$ was performed according to the general procedure for cyclization $\left(150{ }^{\circ} \mathrm{C}\right.$, $36 \mathrm{~h})$ to afford 9 as a colorless oil (93 $\mathrm{mg}, 58 \%)$ after hydrolysis and silica gel chromatography (4\% EtOAc/hex). IR: 1696, 1587, 1248, $1112 \mathrm{~cm}^{-1} \cdot{ }^{1} \mathrm{H}$ NMR (400 MHz): $\delta 10.13(\mathrm{~s}, 1 \mathrm{H}), 7.61(\mathrm{~d}, 1 \mathrm{H}, J=7.5 \mathrm{~Hz}), 7.59-7.56(\mathrm{~m}, 2 \mathrm{H}), 7.43(\mathrm{~d}, 1 \mathrm{H}, J=7.3$ Hz), 7.40-7.37 (m, 3H), $7.30(\mathrm{t}, 1 \mathrm{H}, J=7.5 \mathrm{~Hz}), 3.56(\mathrm{dd}, 1 \mathrm{H}, J=17.3,9.0 \mathrm{~Hz}), 3.12-$ $3.01(\mathrm{~m}, 2 \mathrm{H}), 2.84(\mathrm{dd}, 1 \mathrm{H}, J=15.9,10.9 \mathrm{~Hz}), 1.98-1.86(\mathrm{~m}, 1 \mathrm{H}), 0.36(\mathrm{~s}, 3 \mathrm{H}), 0.35(\mathrm{~s}$, 3H). ${ }^{13} \mathrm{C}$ NMR $(100 \mathrm{MHz}): \delta 192.8,146.9,146.8,137.9,133.9,132.1,129.6,129.4$, 129.1, 127.8, 126.6, 34.1, 33.8, 25.6, -4.38, -4.71. EI-HRMS Calcd. for $\mathrm{C}_{18} \mathrm{H}_{20} \mathrm{OSi}[\mathrm{M}]^{+}$ 280.1283; Found, 280.1278.

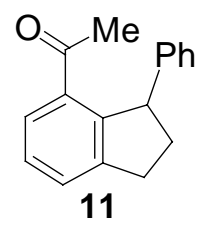

1-(3-Phenyl-indan-4-yl)-ethanone (11). Reaction of $\mathbf{1 0}$ (100 mg, $0.307 \mathrm{mmol})$ was performed according to the general procedure for cyclization $\left(150{ }^{\circ} \mathrm{C}, 16 \mathrm{~h}\right)$ to afford 11 as a white solid (50 mg, 68\% yield) after hydrolysis and silica gel chromatography (10\% EtOAc/Hex). Mp 30-32 ${ }^{\circ} \mathrm{C} . \quad$ IR: $2951,1684,1258,701 \mathrm{~cm}^{-1} .{ }^{1} \mathrm{H}$ NMR (400 MHz): $\delta 7.64(\mathrm{~d}, J=7.6 \mathrm{~Hz}, 1 \mathrm{H}), 7.50(\mathrm{~d}, J=7.2 \mathrm{~Hz}, 1 \mathrm{H}), 7.35(\mathrm{t}, J=7.6 \mathrm{~Hz}, 1 \mathrm{H}), 7.21$ 
(m, 2H), $7.14(\mathrm{~m}, 1 \mathrm{H}), 6.94(\mathrm{~m}, 2 \mathrm{H}), 5.02(\mathrm{dd}, J=2.8,8.8 \mathrm{~Hz}, 1 \mathrm{H}), 3.11-3.07(\mathrm{~m}, 1 \mathrm{H})$, $2.93(\mathrm{dd}, J=3.6,8.8 \mathrm{~Hz}, 1 \mathrm{H}), 2.65-2.56(\mathrm{~m}, 1 \mathrm{H}), 2.35(\mathrm{~s}, 3 \mathrm{H}), 2.10-2.04(\mathrm{~m}, 1 \mathrm{H})$.

${ }^{13} \mathrm{C}\{\mathrm{H}\}$ NMR (125 MHz): $\delta 199.8,146.7,146.3,145.9,135.1,128.4,128.2,127.7$, 127.2, 127.1, 125.7, 50.7, 35.8, 30.8, 28.4. EI-HRMS, Calcd. for $\mathrm{C}_{17} \mathrm{H}_{16} \mathrm{O}[\mathrm{M}]^{+}$ 236.1205; Found, 236.1201.

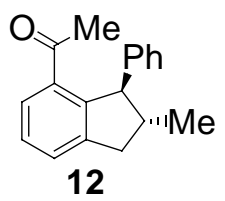

1-(2-Methyl-3-phenyl-indan-4-yl)-ethanone (13). Reaction of $12(150 \mathrm{mg}$, $0.442 \mathrm{mmol})$ was performed according to the general procedure for cyclization $\left(150{ }^{\circ} \mathrm{C}\right.$, $36 \mathrm{~h}$ ) to afford a 7:1 mixture of trans:cis $\mathbf{1 3}$ as a white solid (55 $\mathrm{mg}, 50 \%$ yield) after hydrolysis and silica gel chromatography (5\% EtOAc/Hex). Recystallization from hexanes/Et ${ }_{2} \mathrm{O}$ yielded a trans:cis ratio of 19:1. Further recrystallization by vapor diffusion yielded X-ray quality crystals. The trans isomer of $\mathbf{1 3}$ was determined to be the major isomer by both an X-ray structure of these crystals and a NOESY spectrum of the 7:1 diastereomer mixture. IR: $1671,1581,1430,1250 \mathrm{~cm}^{-1}$. trans: ${ }^{1} \mathrm{H}$ NMR $(500$ MHz): $\delta 7.58(\mathrm{~d}, J=7.6 \mathrm{~Hz}, 1 \mathrm{H}), 7.47(\mathrm{~d}, J=7.3 \mathrm{~Hz}, 1 \mathrm{H}), 7.34(\mathrm{t}, J=7.6 \mathrm{~Hz}, 1 \mathrm{H}), 7.28-$ $7.14(\mathrm{~m}, 3 \mathrm{H}), 6.98(\mathrm{~d}, J=7.1 \mathrm{~Hz}, 2 \mathrm{H}), 4.60(\mathrm{~d}, J=4.8 \mathrm{~Hz}, 1 \mathrm{H}), 3.26(\mathrm{dd}, J=7.6,15.9$ $\mathrm{Hz}, 1 \mathrm{H}), 2.59(\mathrm{dd}, J=5.6,15.8 \mathrm{~Hz}, 1 \mathrm{H}), 2.52-2.41(\mathrm{~m}, 1 \mathrm{H}), 2.28$ (s, 3H), 1.19 (d, $J=6.9$ Hz, 1H). cis: ${ }^{1} \mathrm{H}$ NMR (500 MHz): $\delta 7.69(\mathrm{~d}, J=7.7 \mathrm{~Hz}, 1 \mathrm{H}), 7.48(\mathrm{~d}, J=8.0 \mathrm{~Hz}, 1 \mathrm{H})$, 7.35-7.12 (m, 4H), $6.83(\mathrm{~d}, J=7.5 \mathrm{~Hz}, 2 \mathrm{H}), 4.91(\mathrm{~d}, \mathrm{~J}=8.5 \mathrm{~Hz}, 1 \mathrm{H}), 2.95(\mathrm{dd}, J=7.0$, $15 \mathrm{~Hz}, 1 \mathrm{H}), 2.84-2.88(\mathrm{~m}, 1 \mathrm{H}), 2.69(\mathrm{~m}, 1 \mathrm{H}), 2.38(\mathrm{~s}, 3 \mathrm{H}), 0.78(\mathrm{~d}, \mathrm{~J}=7.0 \mathrm{~Hz}, 3 \mathrm{H})$. trans: ${ }^{13} \mathrm{C}\{\mathrm{H}\}$ NMR $(125 \mathrm{MHz}): \delta 200.2,145.8,145.5,145.4,136.1,128.3,128.2,127.4$, 
127.3, 127.1, 125.8, 59.3, 44.5, 39.2, 28.5, 20.6. HRMS (EI+) Calcd. for $\mathrm{C}_{18} \mathrm{H}_{18} \mathrm{O}[\mathrm{M}]^{+}$ 250.1358; Found, 250.1359.

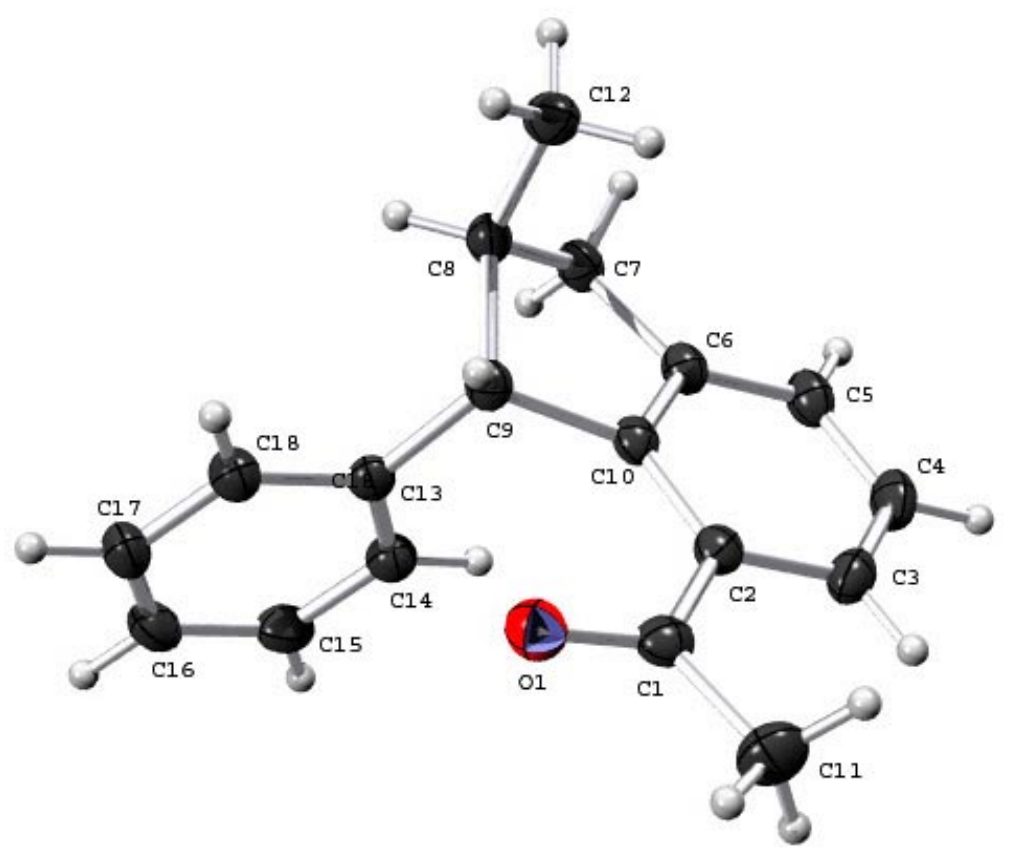

Figure 1. ORTEP drawing of 13. Thermal ellipsoids are shown at the 50\% probability level.

2D NOESY spectrum of 13: A gradient 2D NOESY spectrum of $\mathbf{1 3}$ was acquired in $\mathrm{CDCl}_{3}$ at $22.3{ }^{\circ} \mathrm{C}$ on a Bruker DRX-500 spectrometer using a $5 \mathrm{~mm}$ zBBO (broad band with z-gradient) probe. The pulse program noesygpph was used to generate a phasesensitive spectrum with TPPI phase-cycling. The $\pi / 2$ pulse length was calibrated and found to be $12.20 \mu \mathrm{sec}$. Based on measured $\mathrm{T}_{1}$ 's, the data were collected with a mixing time of 2.0 seconds and a recycle delay of 5.4 seconds. Data were centered at $\mathrm{O} 1=4.0$ ppm and spread over a sweep width of $8.0 \mathrm{ppm}$. The spectrum was acquired in 4 scans per increment, in 200 increments (F1) with 2048 data points (F2). Zero-filling to $512 \mathrm{x}$ 2048 and squared cosine multiplication of both dimensions were applied to the data prior 
to Fourier transformation. The resulting base planes were corrected by applying the Bruker $a b s$ routine, using a $3^{\text {rd }}$ order polynomial fit in both dimensions.

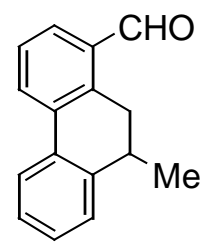

22

9-Methyl-9,10-dihydro-phenanthrene-1-carbaldehyde (22). Reaction of 21 (187 $\mathrm{mg}, 0.601 \mathrm{mmol}$ ) was performed according to the general procedure for cyclization $\left(150{ }^{\circ} \mathrm{C}, 6 \mathrm{~h}\right)$ to afford 22 as a colorless oil (109 $\left.\mathrm{mg}, 81 \%\right)$ after hydrolysis and silica gel chromatography (4\% EtOAc/hex). IR: 1694, $757 \mathrm{~cm}^{-1} .{ }^{1} \mathrm{H}$ NMR (400 MHz): $\delta 10.38$ (s, $1 \mathrm{H}), 8.02(\mathrm{dd}, J=7.8,0.9 \mathrm{~Hz}, 1 \mathrm{H}), 7.80(\mathrm{dd}, J=7.7,1.3 \mathrm{~Hz}, 1 \mathrm{H}), 7.76-7.74(\mathrm{~m}, 1 \mathrm{H})$, $7.49(\mathrm{t}, J=7.7 \mathrm{~Hz}, 1 \mathrm{H}), 7.37-7.33(\mathrm{~m}, 3 \mathrm{H}), 3.41(\mathrm{dd}, J=16.1,5.4 \mathrm{~Hz}, 1 \mathrm{H}), 3.33$ (dd, $J=$ 16.1, 7.2 Hz, 1H), 3.11-3.03 (m, 1H), $1.25(\mathrm{~d}, J=7.0 \mathrm{~Hz}, 3 \mathrm{H}) .{ }^{13} \mathrm{C}$ NMR $(100 \mathrm{MHz}): \delta$ $192.8,141.8,138.6,135.7,134.1,132.6,131.3,129.2,128.4,127.0,126.8,126.4,124.3$, 32.1, 31.0, 19.5. Anal. Calcd. for $\mathrm{C}_{16} \mathrm{H}_{14} \mathrm{O}$ : C, 86.45; H, 6.35. Found: C, 86.22; H, 6.64.<smiles>CC(=O)CC1COc2cccc(C=O)c21</smiles>

30

(4-Formyl-2,3-dihydro-benzofuran-3-yl)-acetic acid methyl ester (30).

Reaction of 29 (740 $\mathrm{mg}, 2.39 \mathrm{mmol})$ was performed according to the general procedure for cyclization $\left(150{ }^{\circ} \mathrm{C}, 48 \mathrm{~h}\right)$ to afford $\mathbf{3 0}$ as a colorless oil $(265 \mathrm{mg}, 50 \%)$ after hydrolysis and silica gel chromatography (15\% EtOAc/hex). IR: 2956, 2897, 2826, $2738,1735,1692,1590,1453,1246,1208,1173,789,766 \mathrm{~cm}^{-1} .{ }^{1} \mathrm{H}$ NMR (400 MHz): $\delta 10.0(\mathrm{~s}, 1 \mathrm{H}), 7.39-7.33(\mathrm{~m}, 2 \mathrm{H}), 7.06(\mathrm{dd}, J=7.2,1.8 \mathrm{~Hz}, 1 \mathrm{H}), 4.69-4.64(\mathrm{~m}, 1 \mathrm{H}), 4.53$ 
(dd, $J=9.4,3.2 \mathrm{~Hz}, 1 \mathrm{H}), 4.28-4.22(\mathrm{~m}, 1 \mathrm{H}), 3.70(\mathrm{~s}, 3 \mathrm{H}), 2.93(\mathrm{dd}, J=16.7,3.1 \mathrm{~Hz}, 1 \mathrm{H})$,

$2.47(\mathrm{dd}, J=16.7,10.9 \mathrm{~Hz}, 1 \mathrm{H}) .{ }^{13} \mathrm{C}$ NMR $(100 \mathrm{MHz}): \delta 192.4,172.3,172.1,161.1$, 132.9, 129.3, 129.2, 126.0, 115.5, 51.7, 38.0, 37.6. Anal. Calcd. for $\mathrm{C}_{12} \mathrm{H}_{12} \mathrm{O}_{4}$ : C, 65.45; H, 5.49. Found: 65.45; H, 5.54.

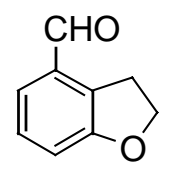

32

2,3-Dihydro-benzofuran-4-carbaldehyde (32). Reaction of $\mathbf{3 1}$ (715 mg, 3.01 mmol) was performed according to the general procedure for cyclization $\left(150{ }^{\circ} \mathrm{C}, 12 \mathrm{~h}\right)$ to afford $\mathbf{3 2}$ as a yellow oil (290 $\mathrm{mg}, 65 \%$ ) after hydrolysis and silica gel chromatography (5\% EtOAc/hex). IR: 2973, 2902, 2822, 2731, 1692, 1592, 1482, 1458, 1394, 1331, 1242, 1215, 982, $778 \mathrm{~cm}^{-1} .{ }^{1} \mathrm{H}$ NMR (400 MHz): $\delta 10.05$ (s, 1H), 7.32-7.29 (m, 2H), 7.04-7.00 (m, 1H), $4.65(\mathrm{t}, J=8.8 \mathrm{~Hz}, 2 \mathrm{H}), 3.54(\mathrm{t}, J=8.8 \mathrm{~Hz}, 2 \mathrm{H}) .{ }^{13} \mathrm{C}$ NMR $(100$ MHz): $\delta 192.4,161.2,132.9,128.5,127.9,124.2,114.7,72.1,29.4$. Anal. Calcd. for $\mathrm{C}_{9} \mathrm{H}_{8} \mathrm{O}_{2}:$ C, 72.96; H, 5.44. Found: C, 73.23; H, 5.69.

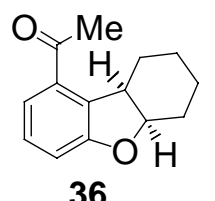

1-(5a,6,7,8,9,9a-Hexahydro-dibenzofuran-1-yl)-ethanone (36). Reaction of 35 (400 $\mathrm{mg}, 1.31 \mathrm{mmol}$ ) was performed according to the general procedure for cyclization $\left(150{ }^{\circ} \mathrm{C}, 72 \mathrm{~h}\right)$ to afford 36 as a colorless oil $(116 \mathrm{mg}, 41 \%)$ after hydrolysis and silica gel chromatography (5\% MTBE/hex). The product was determined to have a cis ring fusion by a 2D NOESY experiment. IR: 2925, 2848, 1679, 1438, 1258, $1217 \mathrm{~cm}^{-1} \cdot{ }^{1} \mathrm{H}$ NMR (500 MHz): $\delta 7.36(\mathrm{~d}, J=7.5 \mathrm{~Hz}, 1 \mathrm{H}), 7.20(\mathrm{t}, J=8.0 \mathrm{~Hz}, 1 \mathrm{H}), 7.01(\mathrm{~d}, J=7.01 \mathrm{~Hz}, 1 \mathrm{H})$, 
$4.56(\mathrm{~m}, 1 \mathrm{H}), 3.62(\mathrm{dt}, J=11.5,6.0 \mathrm{~Hz}, 1 \mathrm{H}), 2.58(\mathrm{~s}, 3 \mathrm{H}), 2.31(\mathrm{br} \mathrm{d}, J=15 \mathrm{~Hz}, 1 \mathrm{H})$, 2.14-2.12 (m, 1H), 1.79-1.72 (m, 1H), 1.65-1.62 (m, 2H), 1.52-1.47 (m, 1H), 1.31-1.25 (m, 1H), 0.96-0.88 (m, 1H). ${ }^{13} \mathrm{C}$ NMR (125 MHz): $\delta 198.9,160.3,136.1,133.2,127.6$, 121.9, 114.4, 83.3, 40.9, 28.0, 27.9, 27.2, 23.0, 20.4. EI-HRMS Calcd. for $\mathrm{C}_{14} \mathrm{H}_{16} \mathrm{O}_{2}$ $[M]^{+}:$216.1150. Found: 216.1148. 2D NOESY spectrum of 36: A gradient 2D NOESY spectrum of $\mathbf{3 6}$ was acquired using the same parameters as those used to obtain a $2 \mathrm{D}$ NOESY spectrum of ketone 13 , except that the acquisition temperature was $23.6{ }^{\circ} \mathrm{C}$ and a recycle delay of 3.25 seconds was used.

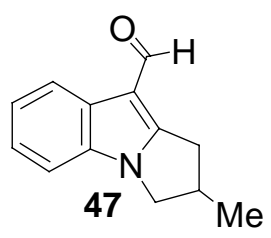

\section{2-Methyl-2,3-dihydro-1H-3a-aza-cyclopenta[a]indene-8-carbaldehyde (47).}

Reaction of 45 (200 mg, $0.694 \mathrm{mmol}$ ) was performed according to the general procedure for cyclization $\left(125^{\circ} \mathrm{C}, 17 \mathrm{~h}\right)$ except that the product was hydrolyzed on silica gel instead of using $1 \mathrm{~N} \mathrm{HCl} .^{11}$ Thus, after the cyclization was complete, the reaction solvent was removed in vacuo and the crude imine product was eluted on silica gel (30\% EtOAc/Hex) to afford aldehyde 47 as a white solid (134 mg, 97\% yield). Mp 116-119 ${ }^{\circ} \mathrm{C}$. IR: 1640, 1436, $1041 \mathrm{~cm}^{-1} . \quad{ }^{1} \mathrm{H}$ NMR $(500 \mathrm{MHz}): \delta 9.98(\mathrm{~s}, 1 \mathrm{H}), 8.29(\mathrm{~d}, J=7.5 \mathrm{~Hz}, 1 \mathrm{H}), 7.28-$ $7.22(\mathrm{~m}, 3 \mathrm{H}), 4.26(\mathrm{dd}, J=8.0,10.5 \mathrm{~Hz}, 1 \mathrm{H}), 3.67(\mathrm{dd}, J=6.5,10.5 \mathrm{~Hz} 1 \mathrm{H}), 3.45(\mathrm{dd}, J$ $=8.5,17.0 \mathrm{~Hz}, 1 \mathrm{H}), 3.24-3.14(\mathrm{~m}, 1 \mathrm{H}), 2.86(\mathrm{dd}, J=7.0,17.0 \mathrm{~Hz}, 1 \mathrm{H}), 1.34(\mathrm{~d}, J=7.0$ $\mathrm{Hz}, 3 \mathrm{H}) .{ }^{13} \mathrm{C}\{\mathrm{H}\} \mathrm{NMR}(125 \mathrm{MHz}): \delta 183.4,155.1,133.2,129.5,122.8,122.6,121.4$, 110.4, 109.9, 51.6, 36.5, 32.8, 19.6. EI-HRMS Calcd. for $\mathrm{C}_{13} \mathrm{H}_{13} \mathrm{NO}[\mathrm{M}]^{+}$199.0995; Found, 199.0997. 


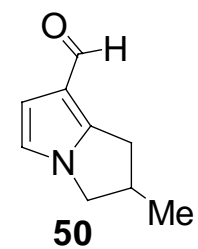

6-Methyl-6,7-dihydro-5H-pyrrolizine-1-carbaldehyde (50). Reaction of 49 (180 $\mathrm{mg}, 0.755 \mathrm{mmol}$ ) was performed according to the general procedure for cyclization $\left(150{ }^{\circ} \mathrm{C}, 60 \mathrm{~h}\right)$ except that the product was hydrolyzed on silica gel instead of using $1 \mathrm{~N}$ $\mathrm{HCl}^{11}$ Thus, after the cyclization was complete, the reaction solvent was removed in vacuo and the crude imine product was eluted on silica gel (50\% EtOAc/Hex) to afford $\mathbf{5 0}$ as an orange-brown oil (83 $\mathrm{mg}, 74 \%$ yield). IR: 2962, 1650, 1536, 1434, 1270, 711 $\mathrm{cm}^{-1} . \quad{ }^{1} \mathrm{H}$ NMR $(500 \mathrm{MHz}): \delta 9.69(\mathrm{~s}, 1 \mathrm{H}), 7.26(\mathrm{~m}, 1 \mathrm{H}), 6.61(\mathrm{~d}, J=3 \mathrm{~Hz}, 1 \mathrm{H}), 6.56(\mathrm{~d}$, $J=3 \mathrm{~Hz}, 1 \mathrm{H}), 4.10(\mathrm{dd}, J=7.5,10.5 \mathrm{~Hz}, 1 \mathrm{H}), 3.55(\mathrm{dd}, J=6.5,10.5 \mathrm{~Hz}, 1 \mathrm{H}), 3.27(\mathrm{dd}, J$ $=8.5,16.5 \mathrm{~Hz}, 1 \mathrm{H}), 3.10-3.03(\mathrm{~m}, 1 \mathrm{H}), 2.67(\mathrm{dd}, J=6.5,16.5 \mathrm{~Hz}, 1 \mathrm{H}), 1.26(\mathrm{~d}, J=6.5$ $\mathrm{Hz}, 3 \mathrm{H}) .{ }^{13} \mathrm{C}\{\mathrm{H}\}$ NMR $(125 \mathrm{MHz}): \delta 184.9,117.9,116.3,112.3,112.3,53.8,36.6,32.8$, 19.5. EI-HRMS Calcd. for $\mathrm{C}_{9} \mathrm{H}_{11} \mathrm{NO}[\mathrm{M}]^{+}$149.0841; Found, 149.0839 . 


\section{NMR Spectra}

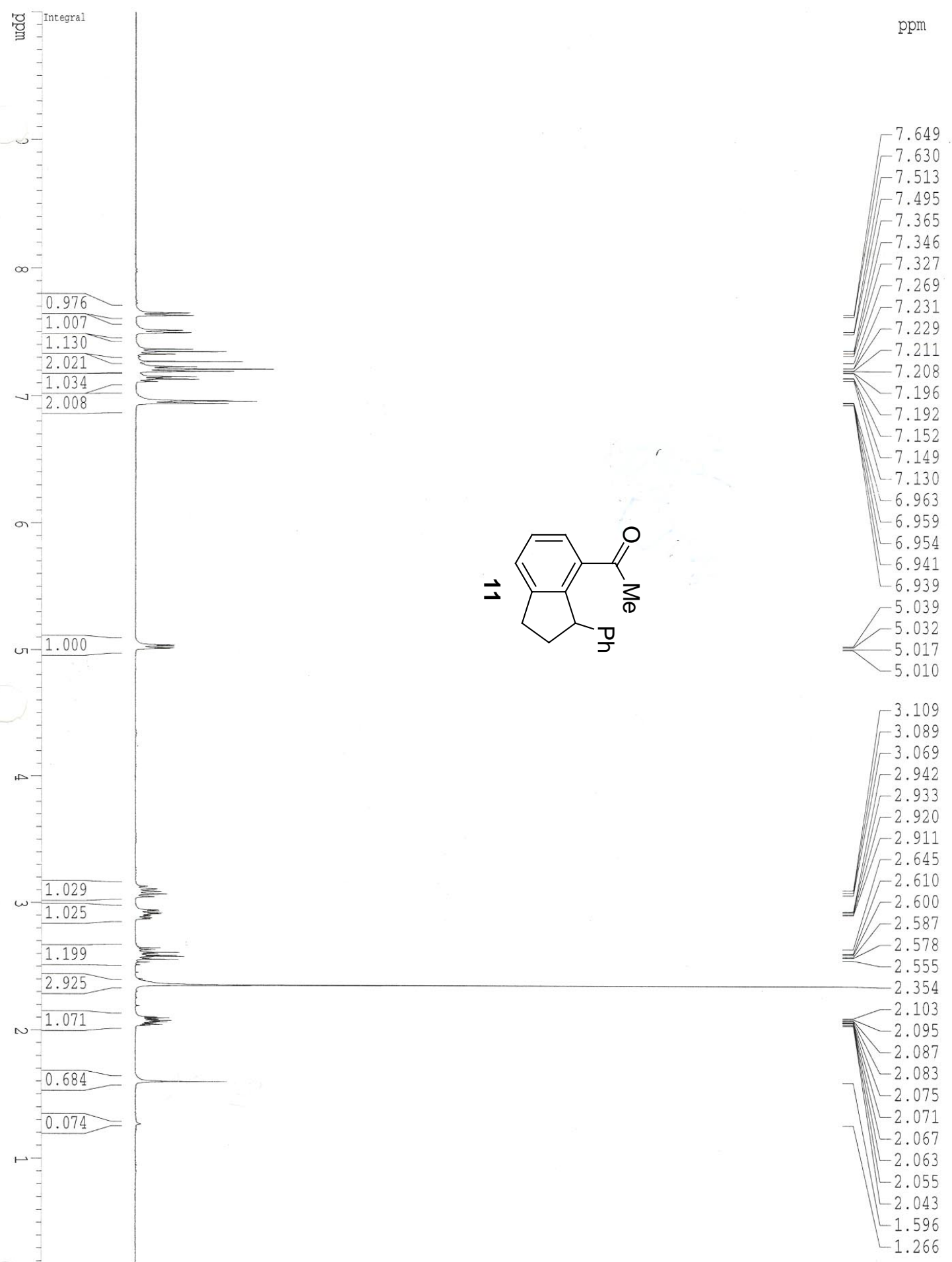




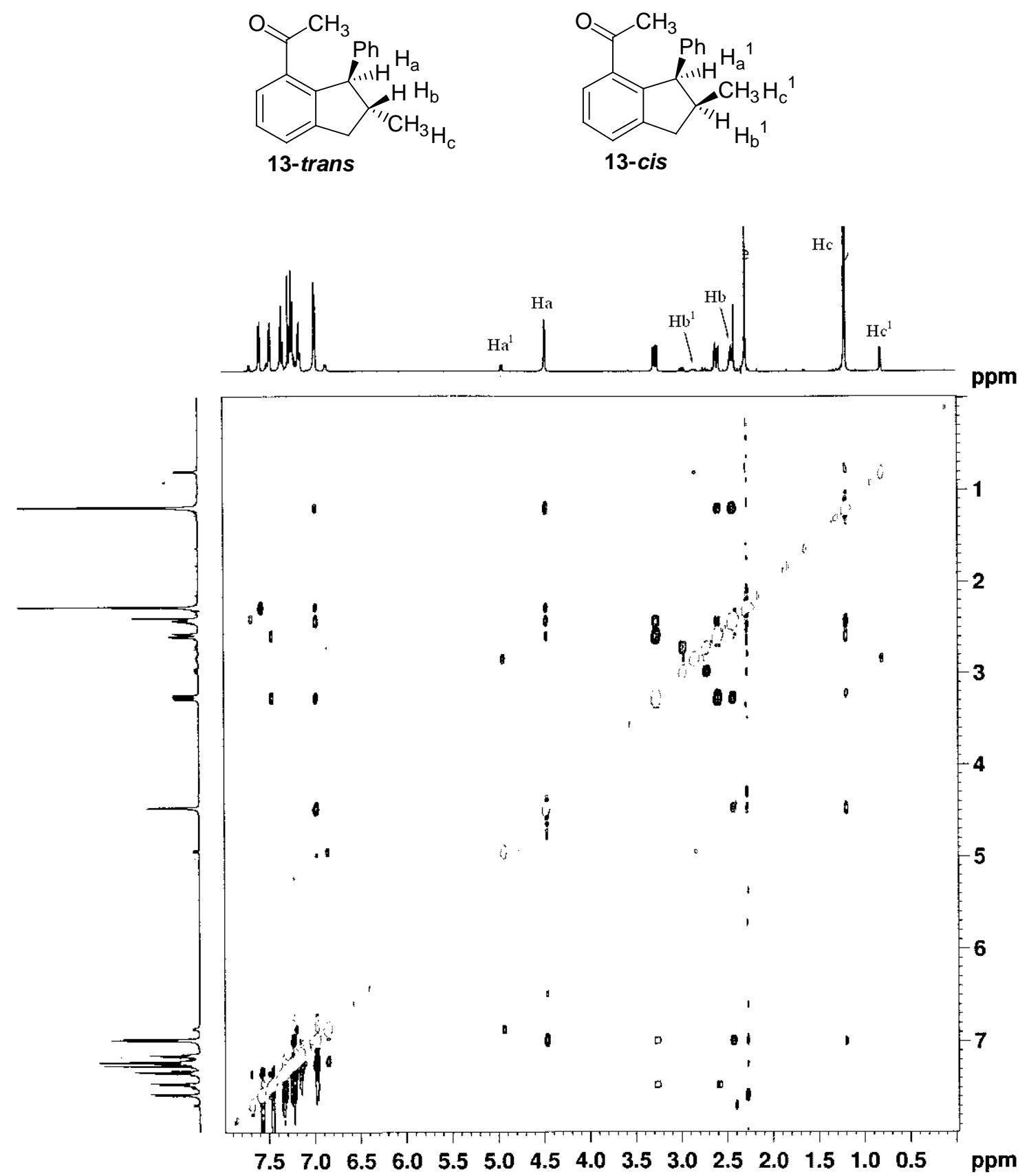

Figure 3. $2 \mathrm{D}{ }^{1} \mathrm{H}-{ }^{1} \mathrm{H}$ NOESY spectrum of 13. NOE between $\mathrm{H}_{\mathrm{a}}$ and $\mathrm{H}_{\mathrm{c}}$ is stronger than that between $\mathrm{H}_{\mathrm{a}}$ and $\mathrm{H}_{\mathrm{b}}$, indicating trans geometry. NOE between $\mathrm{H}_{\mathrm{a}}{ }^{1}$ and $\mathrm{H}_{\mathrm{c}}{ }^{1}$ is weaker than that between $\mathrm{H}_{\mathrm{a}}{ }^{1}$ and $\mathrm{H}_{\mathrm{b}}{ }^{1}$, indicating cis geometry. 


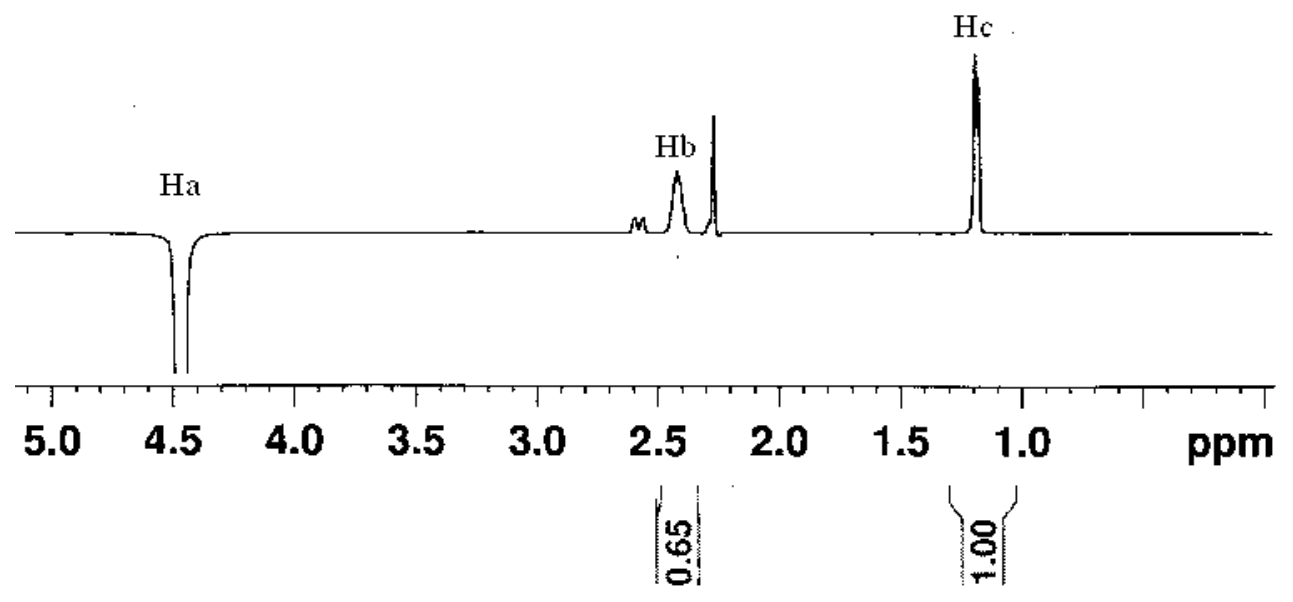

Figure 4. Cross section of the $2 \mathrm{D}{ }^{1} \mathrm{H}^{-}{ }^{1} \mathrm{H}$ NOESY spectrum for $\mathbf{1 3}$ displaying the intensity of the correlations of $\mathrm{H}_{\mathrm{a}}$ with $\mathrm{H}_{\mathrm{b}}$ and $\mathrm{H}_{\mathrm{c}}$.

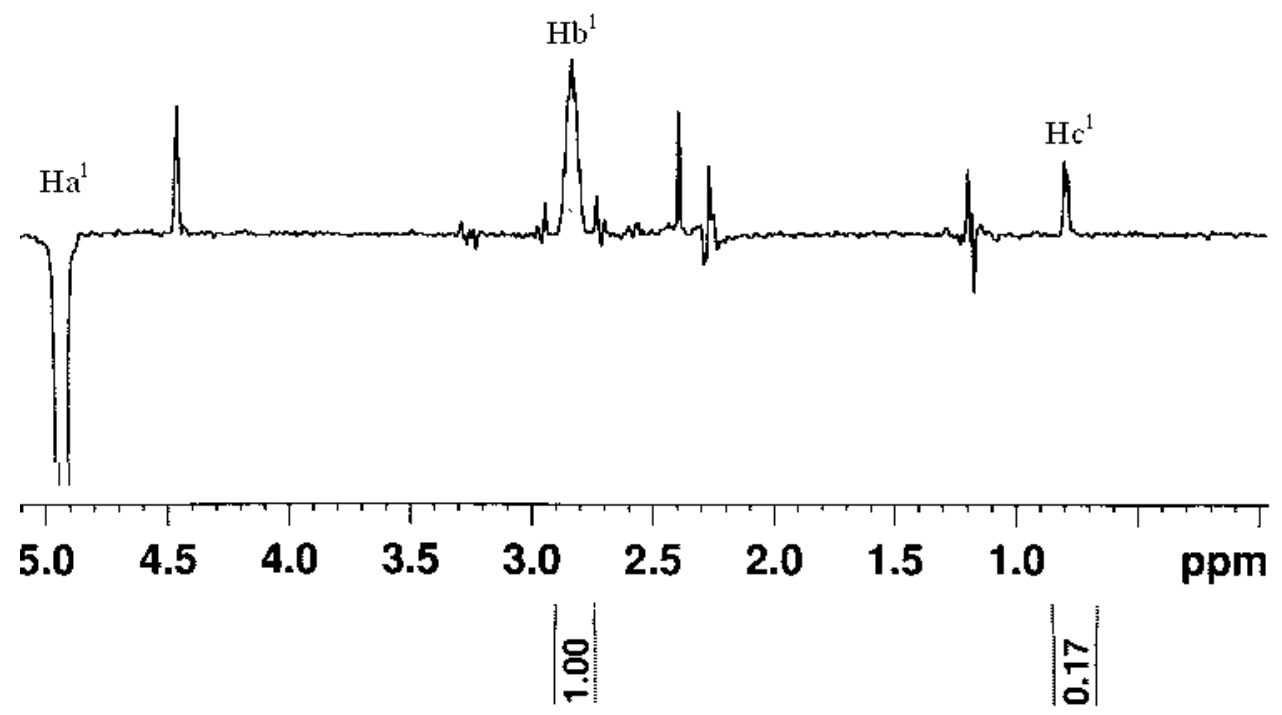

Figure 5. Cross section of the $2 \mathrm{D}{ }^{1} \mathrm{H}-{ }^{1} \mathrm{H}$ NOESY spectrum for $\mathbf{1 3}$ displaying the intensity of the correlations of $\mathrm{H}_{\mathrm{a}}{ }^{1}$ with $\mathrm{H}_{\mathrm{b}}{ }^{1}$ and $\mathrm{H}_{\mathrm{c}}{ }^{1}$. 

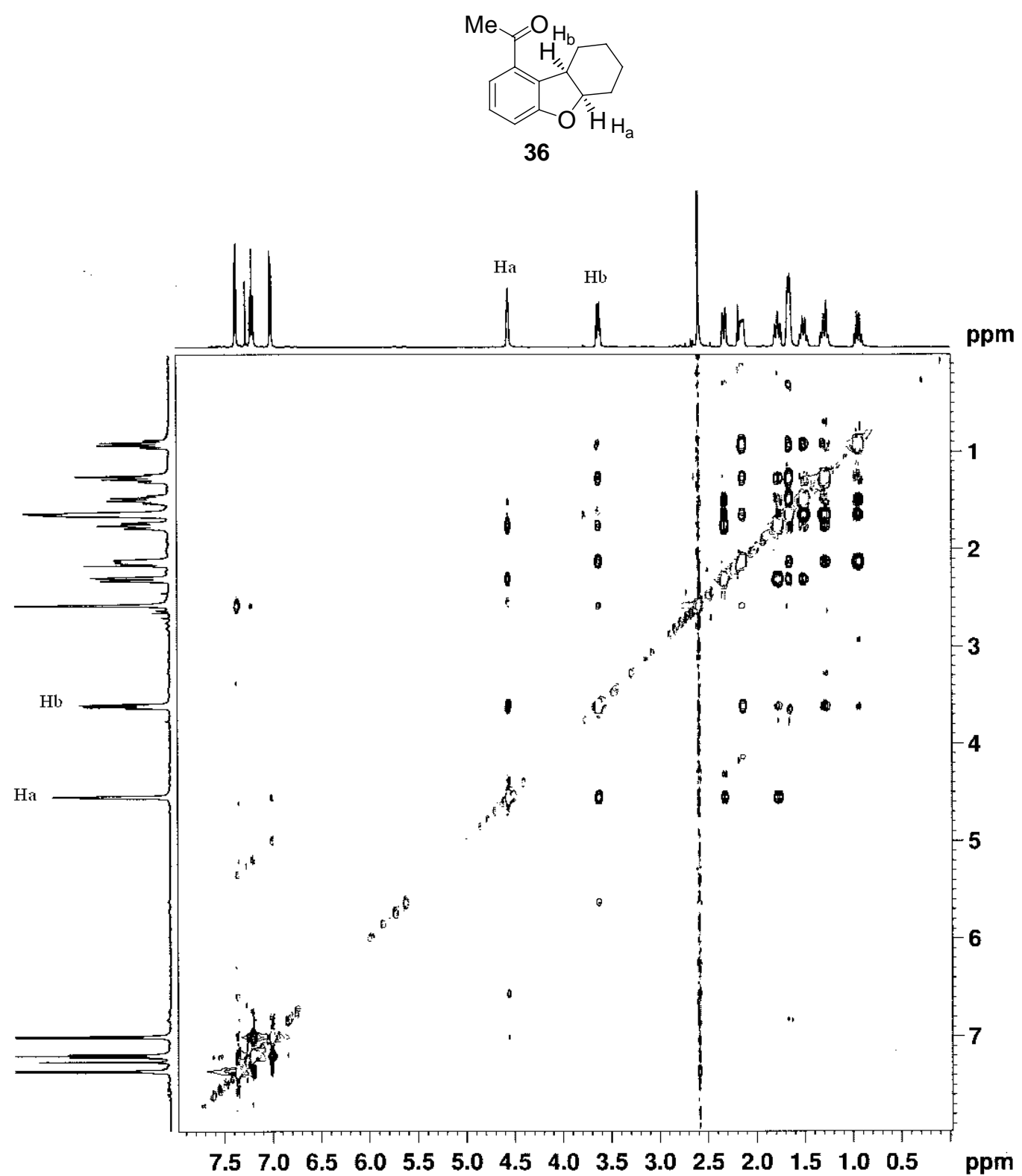

Figure 6. $2 \mathrm{D}{ }^{1} \mathrm{H}-{ }^{1} \mathrm{H}$ NOESY spectrum for 36. Strong NOE correlations between $\mathrm{H}_{\mathrm{a}}$ and $\mathrm{H}_{\mathrm{b}}$ indicate a cis-ring fusion. 


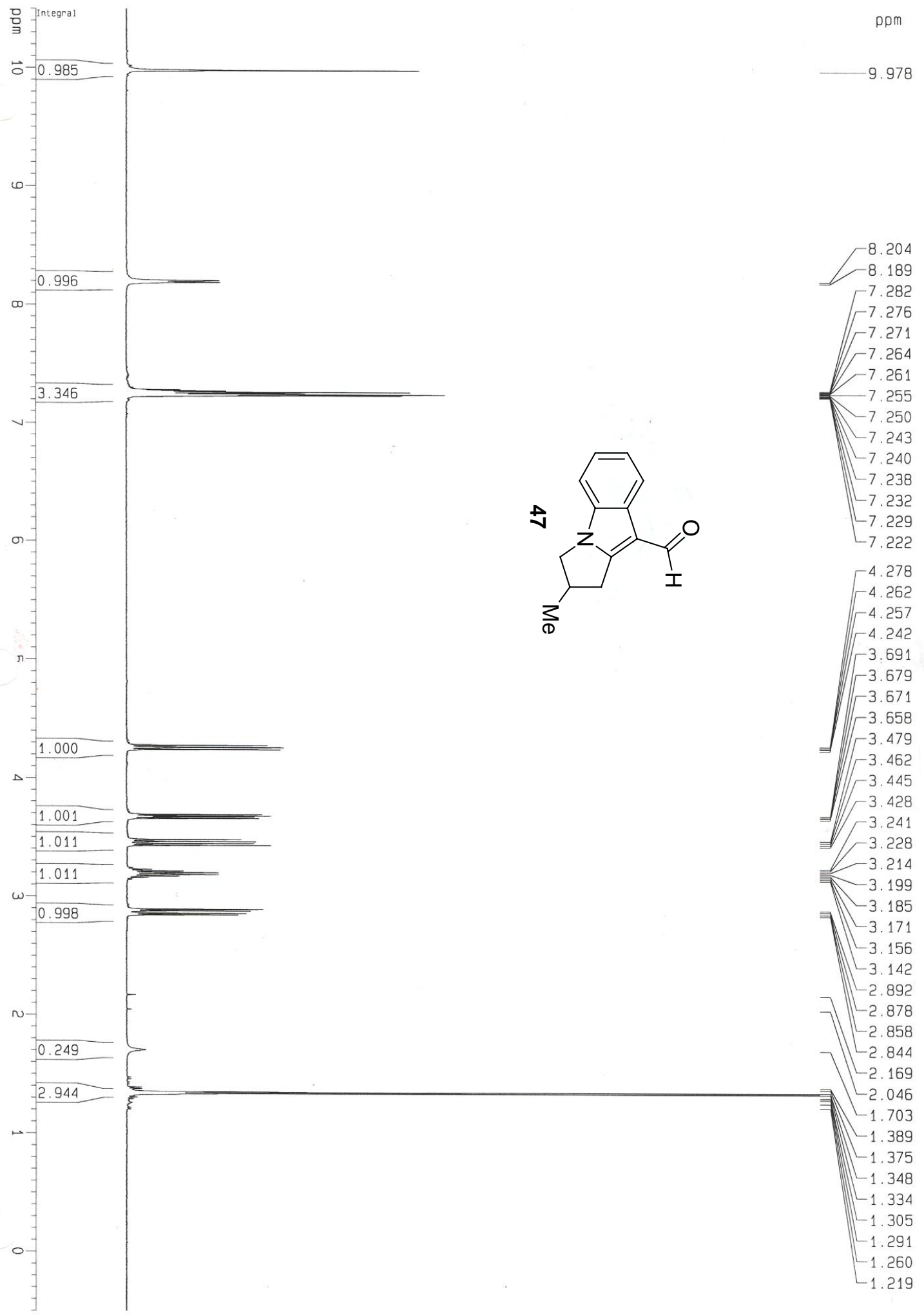




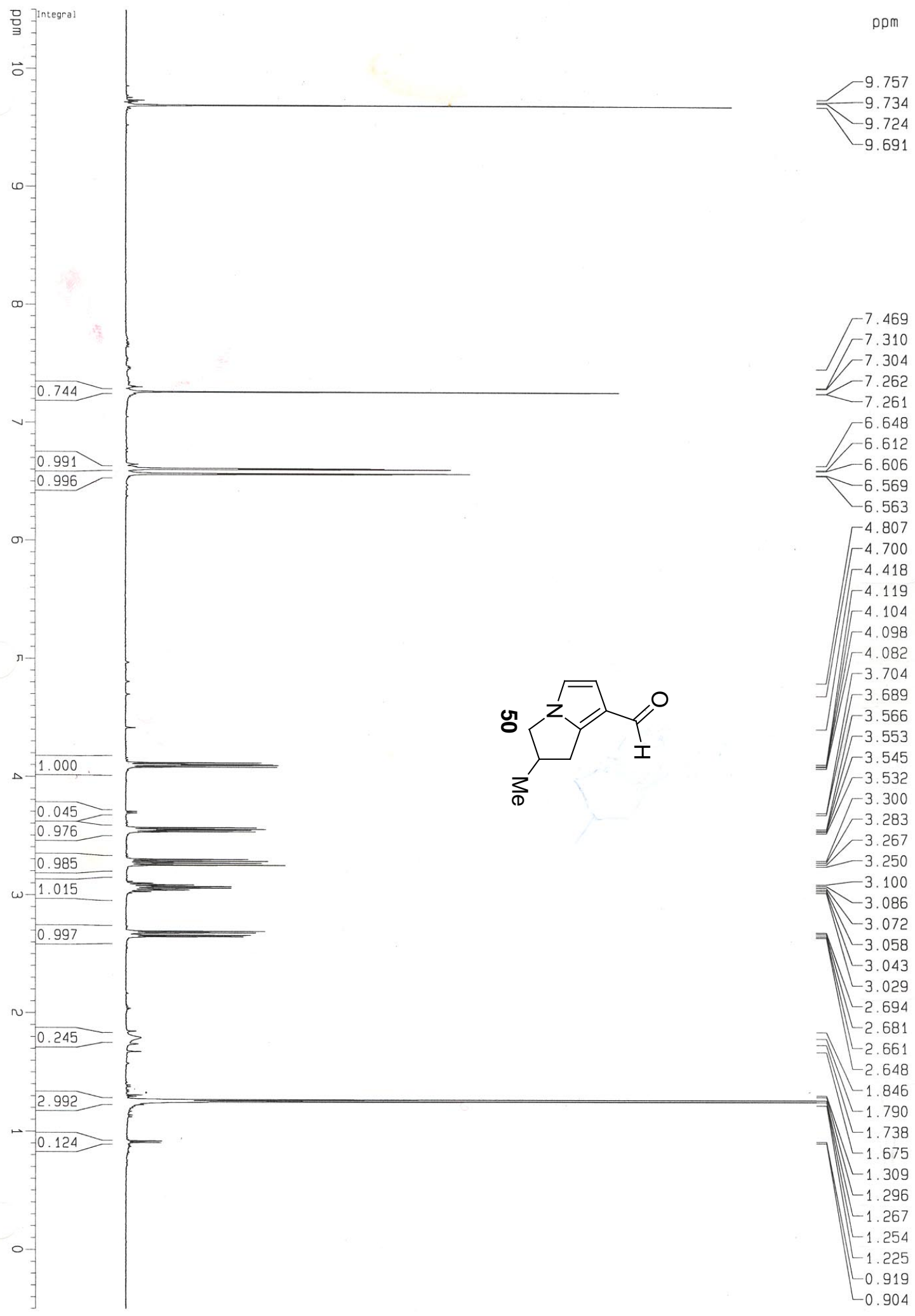




\section{References and Footnotes}

${ }^{1}\left[\mathrm{RhCl}(\mathrm{coe})_{2}\right]_{2}$ is commercially available from Aldrich and Strem, though for these studies it was prepared in one step from $\mathrm{RhCl}_{3}$ and cyclooctene: van der Ent, A.; Onderdelinden, A. L. Inorg. Syn. 1973, 19(14), 1992.

${ }^{2}$ Thalji, R. K.; Ahrendt, K. A.; Bergman, R. G.; Ellman, J. A. J. Am. Chem. Soc. 2001, 123, 9692.

${ }^{3}$ Thalji, R. K.; Bergman, R. G.; Ellman, J. A. J. Am. Chem. Soc. 2004, 126(23), 7192-7193.

${ }^{4}$ Ahrendt, K. A.; Bergman, R. G.; Ellman, J. A. Org. Lett. 2003, 5(8), 1301-1303.

${ }^{5}$ Fleming, I.; Rowley, M.; Cuadrado, P.; Gonzalez-Nogal, A. M.; Pulido, F. J. Tetrahedron 1989, 45, 413.

${ }^{6}$ Prepared as described in Yamakawa, T.; Kagechika, H.; Kawachi, E.; Hashimoto, Y.; Shudo, K. J. Med. Chem. 1990, 33(5), 1430-1437.

${ }^{7}$ Swenton, J. S.; Carpenter, K.; Chen, Y.; Kerns, M. L.; Morrow, G. W. J. Org. Chem. 1993, 53, 3308.

${ }^{8}$ Poradosu, E.; Gazil, A.; Reuveni, H.; Levitzki, A. Bioorg. Med. Chem. 1999, 7, 1727.

${ }^{9}$ Crombie, L.; Jamieson, S. V. J. Chem. Soc. Perkin Trans. I 1982, 1467.

${ }^{10}$ Makela, T.; Matikainen, J.; Wahala, K.; Hase, T. Tetrahedron 2000, 56, 1873

${ }^{11}$ Surprisingly, $1 \mathrm{~N} \mathrm{HCl}$ was not effective in hydrolyzing crude indole- and pyrrole-derived imines. Upon chromatographing these imines, we discovered that, contrary to the benzaldehyde- and acetophenonederived imines, hydrolysis of these imines could be achieved efficiently on silica gel. 